\title{
Farms, Pipes, Streams and Reforestation: Reasoning about Structured Parallel Processes using Types and Hylomorphisms
}

\author{
David Castro, Kevin Hammond, Susmit Sarkar \\ School of Computer Science, University of St Andrews, St Andrews, Scotland \\ $\{$ dc84, kh8, ss265\}@st-andrews.ac.uk
}

\begin{abstract}
The increasing importance of parallelism has motivated the creation of better abstractions for writing parallel software, including structured parallelism using nested algorithmic skeletons. Such approaches provide high-level abstractions that avoid common problems, such as race conditions, and often allow strong cost models to be defined. However, choosing a combination of algorithmic skeletons that yields good parallel speedups for a program on some specific parallel architecture remains a difficult task. In order to achieve this, it is necessary to simultaneously reason both about the costs of different parallel structures and about the semantic equivalences between them. This paper presents a new type-based mechanism that enables strong static reasoning about these properties. We exploit well-known properties of a very general recursion pattern, hylomorphisms, and give a denotational semantics for structured parallel processes in terms of these hylomorphisms. Using our approach, it is possible to determine formally whether it is possible to introduce a desired parallel structure into a program without altering its functional behaviour, and also to choose a version of that parallel structure that minimises some given cost model.
\end{abstract}

Categories and Subject Descriptors D.3.2 [Language Classifications]: Concurrent, distributed, and parallel languages; D.1.3 [Programming Techniques]: Parallel Programming; D.3.1 [Formal Definitions and Theory]: Semantics

Keywords Parallelism, type-systems, hylomorphisms, term rewriting systems.

\section{Introduction}

Providing suitable abstractions to allow reasoning about parallelism is crucial to allow safe exploitation of increasingly parallel hardware. To date, however, there has been only a limited amount of work on this issue: the static analysis community has been concerned primarily with provable safety, whereas the parallelism community has been concerned primarily with practically demonstrable performance. In order to meet both demands, it is necessary to simultaneously consider both the functional and the extra-functional properties of a parallel program. This paper presents a new approach, a type-based mechanism that enables us to reason about the safe introduction of parallelism, while also providing a good abstraction to reason about cost. This mechanism exploits strong program structure in the form of structured parallel processes [3], combined with properties of hylomorphisms [22].

\subsection{Motivating Example}

We introduce our approach using a simple example, image merge, which merges pairs of images taken from an input stream. We start with a simple structure, where the functionality of the program is split into two functions: mark, which marks the pixels that are to be merged; and merge, whichreplaces these pixels. A straightforward implementation would use the usual map construct on lists:

$$
\begin{aligned}
& \text { imgMerge }: \text { List }(\text { Img } \times \text { Img }) \rightarrow \text { List Img } \\
& \text { imgMerge }=\text { map }(\text { merge } \circ \text { mark })
\end{aligned}
$$

Given two well-known algorithmic skeletons, farm for parallel replication and pipeline for parallel composition, even this simple example presents several alternative parallelisations, including:

$$
\begin{aligned}
& \text { imgMerge }_{1}=\text { farm } n \text { (fun (merge } \circ \text { mark)) } \\
& \text { imgMerge }_{2}=\text { farm } n \text { (fun mark) } \| \text { farm } m \text { (fun merge) } \\
& \text { imgMerge }_{3}=\text { farm } n \text { (fun merge) } \| \text { fun mark } \\
& \ldots
\end{aligned}
$$

where $\operatorname{fun}(f)$ encapsulates sequential functionality and $p_{1} \| p_{2}$ represents a 2-stage parallel pipeline. All these implementations are semantically equivalent to imgMerge: they differ only in how the computation is performed, and thus in its performance. In this paper, we will describe a type system that annotates toplevel types with the (parallel) structure, and will use this to $a u$ tomatically choose a parallelisation. For example, if we define $\operatorname{IM}(n, m)=$ FARM $n$ (FUN A) $\|$ FARM $m$ (FUN A), we could write:

$$
\begin{aligned}
& \text { imgMerge : List }(\operatorname{Img} \times \operatorname{Img}) \stackrel{\text { IM }(n, m)}{\longmapsto} \text { List Img } \\
& \text { imgMerge }=\text { map }(\text { merge } \circ \text { mark })
\end{aligned}
$$

The type system will then automatically select imgMerge $e_{2}$. Rather than manually changing the definition of imgMerge to a parallel one, we can simply change the type annotation IM $(n, m)$ and automatically introduce the corresponding parallel implementation. The soundness of the type system provides strong static guarantees that the resulting program is functionally equivalent to the original form. Moreover, we can even use the type system to infer part of this parallel structure. For example, if we define $\operatorname{IM}_{1}(n, m)={ }_{-} \|$ FARM $m_{-}$and $\operatorname{IM}_{2}(n, m)=\min$ cost $\left(-\|\right.$ FARM $\left.m_{-}\right)$, then the parts of $\mathrm{IM}_{1}$ that are denoted by - will be filled with the simplest possible (parallel) structure and the parts of $\mathrm{IM}_{2}$ that are denoted by - will be filled with the least cost (parallel) structure. This provides a powerful, type-level, mechanism for understanding, reasoning about, and automating the introduction of parallelism. 


\subsection{Contributions}

The paper makes the following main novel contributions:

- We define a denotational semantics for a set of well-known algorithmic skeletons, that cover a good range of parallel programs, in terms of hylomorphisms (Section 3).

- We identify semantic equivalences of parallel processes built using nested algorithmic skeletons as part of a type system that allows us to rationally choose a suitable parallel structure for a program (Section 4).

- We introduce a decision procedure for such semantic equivalences, based on reintroducing intermediate data structures, "reforestation", rather than the more common approach of eliminating them for efficiency reasons, "deforestation". This enables the type system to introduce parallelism in a semiautomated and sound way (Section 5).

\section{Type Preliminaries}

Our denotational semantics is phrased in a standard categorical language [10], with a category representing a model of computation, the objects of that category representing types, the morphisms representing programs, and endofunctors from the category to itself representing type constructors. In line with common practice [34], we will work in the category of pointed complete partial orders $(C P O)$. Our type language is entirely standard:

$$
A, B::=\tau|1| A+B|A \times B| A \rightarrow B|F A| \mu F
$$

We assume no knowledge about the definition of an atomic type $(\tau)$, but require it to have an interpretation as an object in the category $C P O, \llbracket \tau \rrbracket \in C P O$. For the other types, we assume a standard domain-theoretic semantics, where types are interpreted as pointed $C P O$ s. The type 1 is interpreted as the unit of $C P O$; the type $A+B$ is interpreted as the separated sum of $\llbracket A \rrbracket$ and $\llbracket B \rrbracket$; the type $A \times B$ is interpreted as the Cartesian product of $\llbracket A \rrbracket$ and $\llbracket B \rrbracket$; the type $A \rightarrow B$ is interpreted as the set of continuous functions from $\llbracket A \rrbracket$ to $\llbracket B \rrbracket$; the type $F A$ is interpreted as the corresponding endofunctor applied to $\llbracket A \rrbracket$; and the type $\mu F$ is interpreted as the fixpoint of functor $\llbracket F \rrbracket$.

\subsection{Functors}

A functor is a structure-preserving mapping between categories. We only need endofunctors on $C P O, F: C P O \rightarrow C P O$, to represent type constructors, $F:$ Type $\rightarrow$ Type. Bifunctors are functors that are generalised to multiple arguments, and we use them to define polymorphic data types. The functors in our language are either standard polynomial functors with constant types, the left section of a bifunctor, or a polymorphic type defined as the fixpoint of some bifunctor. Bifunctors are defined using products and sums alone. Functors are defined using a pointed notation, with the obvious semantic interpretation. If $A$ and $B$ are type variables, and $T$ is a type, we accept the following definitions:

$$
\begin{array}{lr}
G A B=T & \text { (bifunctor defined using sums and products) } \\
F A=T & \text { (functor defined using sums and products) } \\
F A=G T A & (G \text { is a bifunctor) } \\
F A=\mu(G A) & \text { ( } G \text { is a bifunctor) }
\end{array}
$$

Example 1 (Lists). Given the bifunctor

$$
L A B=1+A \times B,
$$

the polymorphic List data type is defined by the fixpoint of $L A$ :

$$
\text { List } A=\mu(L A) \text {. }
$$

As we will discuss in Section 3, it is well known that given a base bifunctor $F A B$, the data type $F A=\mu(G A)$ is also a functor.

$$
\begin{aligned}
& (\cdot \circ):(B \rightarrow C) \rightarrow(A \rightarrow B) \rightarrow A \rightarrow C \\
& f \circ g=\lambda x . f(g x) \\
& (\cdot \nabla \cdot):(A \rightarrow C) \rightarrow(B \rightarrow C) \rightarrow(A+B) \rightarrow C \\
& (f \nabla g)=\lambda x . \text { case } x(\lambda y . f y)(\lambda y . g y) \\
& (\cdot+\cdot):(A \rightarrow C) \rightarrow(B \rightarrow D) \rightarrow(A+B) \rightarrow(C+D) \\
& (f+g)=\left(i n j_{1} \circ f\right) \nabla\left(i n j_{2} \circ g\right) \\
& (\cdot \Delta \cdot):(A \rightarrow B) \rightarrow(A \rightarrow C) \rightarrow A \rightarrow(B \times C) \\
& (f \triangle g)=\lambda x .(f x, g x) \\
& (\cdot \times \cdot):(A \rightarrow C) \rightarrow(B \rightarrow D) \rightarrow(A \times B) \rightarrow(C \times D) \\
& (f \times g)=\left(f \circ \pi_{1}\right) \triangle\left(g \circ \pi_{2}\right)
\end{aligned}
$$

Figure 1: Primitive Combinators.

The two list constructors are defined as expected:

$$
\begin{aligned}
& \text { nil : List } A \\
& \text { nil }=i n_{L A}\left(i n j_{1}()\right) \\
& \text { cons } \quad: \quad A \rightarrow \text { List } A \rightarrow \text { List } A \\
& \text { cons } x l=\operatorname{in}_{L A}\left(i n j_{2}(x, l)\right)
\end{aligned}
$$

We define the usual notation for lists

$$
\left[x_{1}, x_{2}, \ldots, x_{n}\right]=\text { cons } x_{1}\left(\text { cons } x_{2}\left(\text { cons } \ldots\left(\text { cons } x_{n} \text { nil }\right)\right)\right) .
$$

Example 2 (Trees). The polymorphic binary tree type can be defined in an analogous way:

$$
\begin{aligned}
& T A B=1+A \times B \times B \text { where } \\
& \text { Tree } A=\mu(T A) .
\end{aligned}
$$

The two tree constructors are defined below:

$$
\begin{array}{lll}
\text { empty } & : & \text { Tree } A \\
\text { empty } & = & i n_{T A}\left(i n j_{1}()\right) \\
\text { node } & : & \text { Tree } A \rightarrow A \rightarrow \text { Tree } A \rightarrow \text { Tree } A \\
\text { node } t_{1} x t_{2} & =\quad i_{T A}\left(i n j_{2}\left(x, t_{1}, t_{2}\right)\right)
\end{array}
$$

Finally, we use a syntactic notation base $F$ in our typing rules, base $F$ is either the bifunctor $G$ that is used in the definition of $F$, or else $F$ itself.

$$
\text { base } F= \begin{cases}G, & \text { if } F A=G C A \text { or } F A=\mu(G A) \\ F, & \text { otherwise. }\end{cases}
$$

\subsection{Primitive Combinators}

Our semantics is defined in terms of the primitive combinators in Figure 1. For simplicity, we use a flattened form of sum and product types, e.g. we write $A_{1}+A_{2}+\cdots+A_{n}$ rather than $A_{1}+\left(A_{2}+\left(\ldots+A_{n}\right)\right)$. Instead of the usual inl/inr and $\pi_{1} / \pi_{2}$, we use the $i n j_{i}, 0<i \leq n$, introduction forms for sum types, $A_{1}+A_{2}+\cdots+A_{n}$, and the projection eliminators $\pi_{i}, 0<i \leq n$ for product types, $A_{1} \times A_{2} \times \cdots \times A_{n}$. The operator $\circ$ denotes function composition, $\nabla$ is the usual coproduct morphism (join), + denotes the map on coproducts, $\Delta$ and $\times$ are the respective morphisms and maps on products. Finally, for a recursive type defined as the fixpoint of a functor, $\mu F$, the usual $i n_{F}: F \mu F \rightarrow$ $\mu F$ and out $_{F}: \mu F \rightarrow F \mu F$ capture the isomorphism between $F \mu F$ and $\mu F$. 


\section{Structured Parallel Programs}

Structured parallel approaches, such as algorithmic skeletons [3], offer many advantages in terms of built-in safety and parallelismby-construction. For example, they can eliminate by design common but hard-to-debug problems including deadlock and race conditions. Such problems are prevalent in typical low-level concurrency based designs for parallel systems, e.g. pthreads, OpenMP, etc. Algorithmic skeletons also provide good structural cost models $[14,20]$. In this paper, we will use four basic parallel skeletons, each of which operates over a stream of input values, producing a stream of results: task farms, pipelines, feedbacks, and divide-andconquer.

Task farms. The task farm skeleton, farm, applies the same operation to each element of an input stream of tasks, using a fixed number of parallel workers. The input tasks must be independent, and the outputs can be produced in an arbitrary order ${ }^{1}$. For example, a task farm could be used to apply some filter to an input stream of images, in order to parallelise the filter operation.

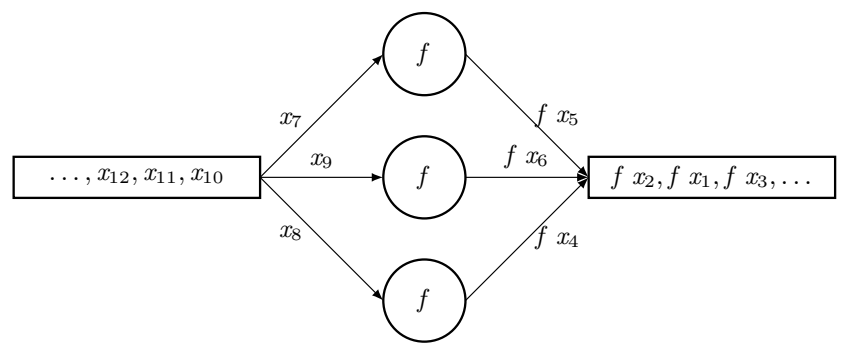

Pipeline. The pipeline skeleton, II, composes two streaming computations, in parallel. It can be used to parallelise two or more stages of a computation, e.g. filtering and edge detection.

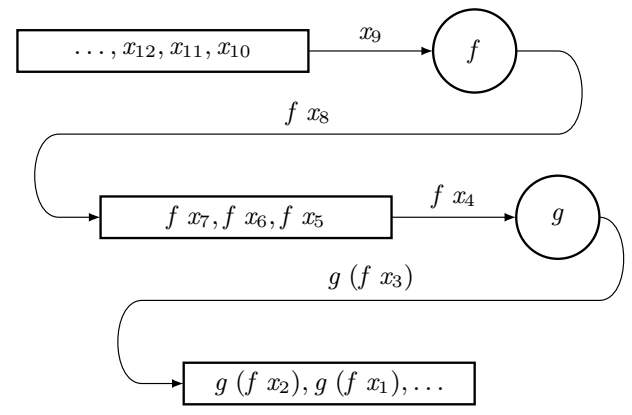

Feedback. The feedback skeleton, fb, captures recursion in a streaming computation. A feedback could be used, for example, to repeatedly transform an image until some dynamic condition was met.

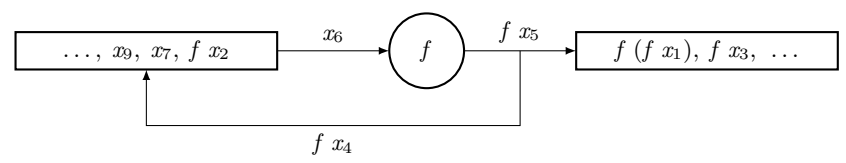

Parallel Divide and Conquer. A divide-and-conquer skeleton, dc, is a parallel implementation of a classical divide-and-conquer algorithm. The parallelism arises from performing each of the recursive calls in parallel.

\footnotetext{
${ }^{1}$ Although this does complicate the semantics, it improves parallelism.
}

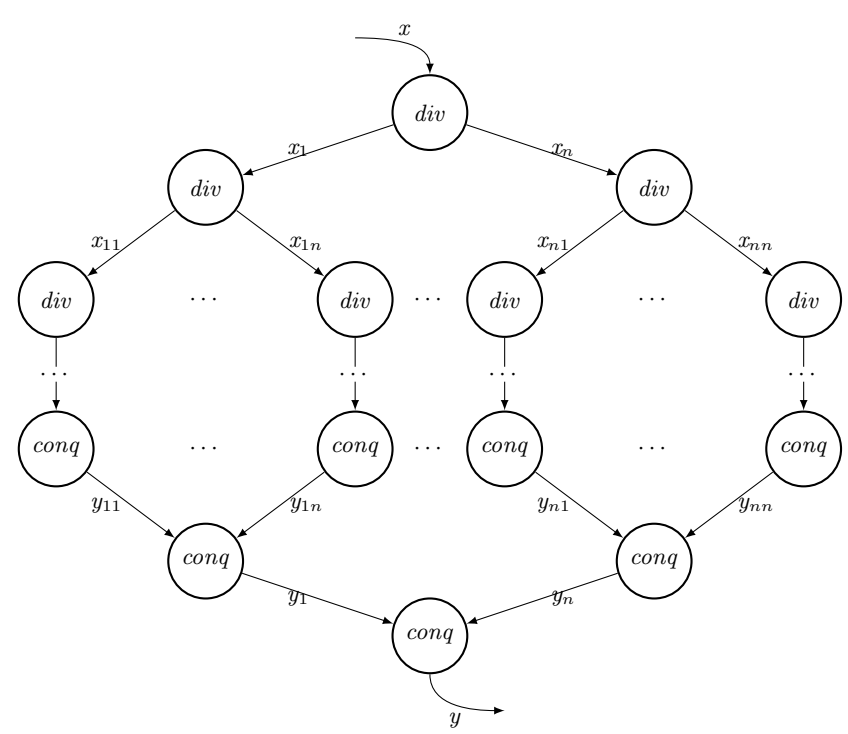

\subsection{Structured Parallel Processes}

We define a language $P$ of structured parallel processes, built by composing skeletons over atomic operations.

$$
p \in P::=\operatorname{fun}_{T} f\left|p_{1} \| p_{2}\right| \mathrm{dc}_{n, T, F} f g \mid \text { farm } n p \mid \text { fb } p
$$

The fun $_{T} f$ construct lifts an atomic function to a streaming operation on a collection $T$. The arguments of the dc skeleton are: the number of levels of the divide-and-conquer, $n$; the collection $T$ on which the dc skeleton works; and the functor $F$ that describes the divide-and-conquer call tree.

Denotational Semantics. The denotational semantics is split into two parts: $\mathcal{S} \llbracket \cdot \rrbracket$ describes the base semantics, and $\llbracket \cdot$ lifts this to a streaming form. We use a global environment for atomic function types, $\rho$, and the corresponding global environment of functions, $\hat{\rho}$ :

$$
\begin{aligned}
& \rho=\{f: A \rightarrow B, \ldots\} \quad \hat{\rho}=\{\llbracket f \rrbracket \in \llbracket A \rightarrow B \rrbracket, \ldots\} \\
& \mathcal{S} \llbracket p: T A \rightarrow T B \rrbracket \quad: \quad \llbracket A \rightarrow B \rrbracket \\
& \mathcal{S} \llbracket \text { fun } f \rrbracket=\hat{\rho}(f) \\
& \mathcal{S} \llbracket p_{1} \quad \| p_{2} \rrbracket \quad=\quad \mathcal{S} \llbracket p_{2} \rrbracket \circ \mathcal{S} \llbracket p_{1} \rrbracket \\
& \mathcal{S} \llbracket \text { farm } n p \rrbracket \quad=\mathcal{S} \llbracket p \rrbracket \\
& \mathcal{S} \llbracket \mathrm{fb} p \rrbracket \quad=\quad \text { iter } \mathcal{S} \llbracket p \rrbracket \\
& \mathcal{S} \llbracket \mathrm{dc}_{n, T, F} f g \rrbracket \quad=\quad \operatorname{cata}_{F}(\hat{\rho}(f)) \circ \operatorname{ana}_{F}(\hat{\rho}(g)) \\
& \begin{array}{ll}
\llbracket p: T A \rightarrow T B \rrbracket \quad: & \llbracket T A \rightarrow T B \rrbracket \\
\llbracket p \rrbracket & \quad \operatorname{map}_{T} \mathcal{S} \llbracket p \rrbracket
\end{array}
\end{aligned}
$$

An atomic function, $f$, is applied to all the elements of a collection of data. A parallel pipeline, $p_{1} \| p_{2}$, is the composition of two parallel processes, $p_{1}$ and $p_{2}$. A task farm, farm $n p$, replicates a parallel process, $p$, so has the same denotational semantics as $p$. A feedback skeleton, fb $p$, applies the computation $p$ iteratively, i.e. trampolined, to the elements in the input collection. Its semantics is given in terms of the function iter.

$$
\begin{aligned}
& \text { iter }:(A \rightarrow A+B) \rightarrow A \rightarrow B \\
& \text { iter } f=\mathrm{Y}(\lambda g \cdot(g \nabla i d) \circ f)
\end{aligned}
$$



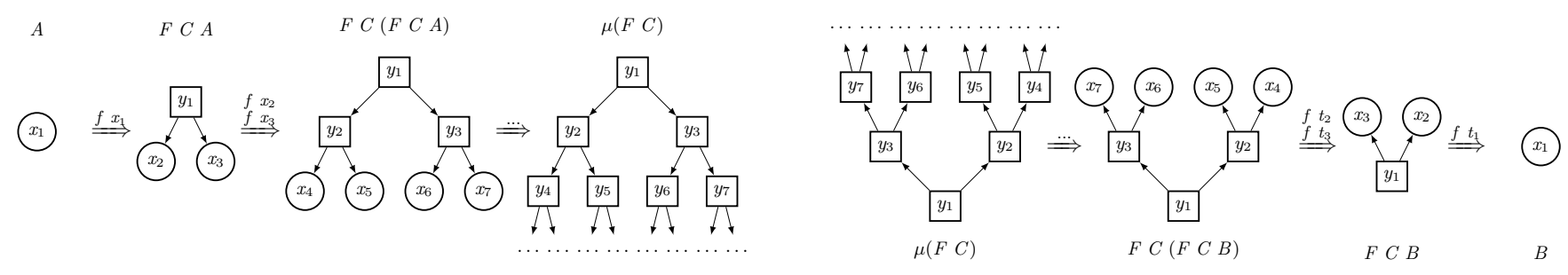

Figure 2: Binary Tree Anamorphism (left) and Catamorphism (right).

Finally, a dc is equivalent to folding, using $f$, the tree-like structure that results from unfolding the input using $g$. It is defined to be the composition of a catamorphism with an anamorphism.

$$
\begin{array}{lll}
\text { cata }_{F} & :(F A \rightarrow A) \rightarrow \mu F \rightarrow A \\
\text { cata }_{F} f & =f \circ F\left(\text { cata }_{F} f\right) \circ \text { out }_{F} \\
\text { ana }_{F} & : \quad(A \rightarrow F A) \rightarrow A \rightarrow \mu F \\
\text { ana }_{F} g= & \text { in }_{F} \circ F\left(\text { ana }_{F} g\right) \circ g
\end{array}
$$

Example 3 (List catamorphism). Let $L$ be the base bifunctor of a polymorphic list. We define the function $f$ to be:

$$
\begin{array}{lll}
f & : & L \mathbb{N} \mathbb{N} \rightarrow \mathbb{N} \\
f\left(\operatorname{inj}_{1}()\right) & = & 0 \\
f\left(\operatorname{inj}_{2}(x, n)\right) & = & \text { add } x n
\end{array}
$$

Given an input list $\left[x_{1}, x_{2}, \ldots, x_{n}\right]$, the catamorphism cata ${ }_{L \mathbb{N}} f$ applied to this input list returns the sum of the $x_{i}$ :

$$
\text { cata }_{L \mathbb{N}} f\left[x_{1}, x_{2}, \ldots, x_{n}\right]=\operatorname{add} x_{1}\left(\operatorname{add} x_{2}\left(\cdots\left(\operatorname{add} x_{n} 0\right)\right)\right) .
$$

Example 4 (List anamorphism). We define a function $g$ that returns () if the input $n$ is zero, and $(n, n-1)$ otherwise.

$$
\begin{array}{lll}
g & : & \mathbb{N} \rightarrow L \mathbb{N} \mathbb{N} \\
g n & = & \text { if } n=0 \text { then } i n j_{1}() \text { else } i n j_{2}(n, n-1)
\end{array}
$$

The anamorphism an $a_{L \mathbb{N}} g$ applied to $n$ returns a list of numbers descending from $n$ to 1 : an $a_{L \mathbb{N}} g n=[n, n-1, \ldots, 2,1]$.

Figure 2 shows how catamorphisms and anamorphisms work on binary trees. In the anamorphism, we start with an input value, and apply the operation $f$ recursively until the entire data structure is unfolded. In the catamorphism, the operation $f$ is applied recursively until the entire structure is folded into a single value. The operation $\operatorname{map}_{T}$ can be defined as a special case of a catamorphism or anamorphism. Given a bifunctor $G$, a type $T A=\mu(G A)$ is a polymorphic data type that is also a functor [10]. For all $f: A \rightarrow B$, the function $\operatorname{map}_{T} f$ is the morphism $T f$, defined as:

$$
\begin{aligned}
\operatorname{map}_{T} f & =\operatorname{cata}_{G A}\left(\text { in }_{G B} \circ G f i d\right) \\
& =\operatorname{ana}_{G B}\left(G f i d \circ \text { out }_{G A}\right)
\end{aligned}
$$

For uniformity, we will represent all $\operatorname{map}_{T}$ as catamorphisms.

Example 5 ( map $\left._{\text {List }}\right)$. Given a function $f: A \rightarrow B, \operatorname{map}_{\text {List }} f$ applies $f$ to all the elements of the input list:

$$
\operatorname{map}_{\text {List }} f\left[x_{1}, x_{2}, \ldots, x_{n}\right]=\left[f x_{1}, f x_{2}, \ldots, f x_{n}\right]
$$

\subsection{Hylomorphisms}

Hylomorphisms are a well known, and very general, recursion pattern [22]. A hylomorphism can be thought of as the generalisation of a divide-and-conquer. Intuitively, $h y l_{F} f g$ is a recursive algorithm whose recursive call tree can be represented by $\mu F$, where $g$ describes how the algorithm divides the input problem into subproblems, and $f$ describes how the results are combined.

$$
\begin{array}{lll}
\text { hylo }_{F} & : & (F B \rightarrow B) \rightarrow(A \rightarrow F A) \rightarrow A \rightarrow B \\
\text { hylo }_{F} f g= & f \circ F(\text { hylo } F g) \circ g
\end{array}
$$

Since out $_{F} \circ i n_{F}=i d$, we can easily show that hylo $f g=$ cata $_{F} f \circ \operatorname{ana}_{F} g$. Catamorphisms, anamorphisms and map are just special cases of hylomorphisms.

$$
\begin{aligned}
& T A=\mu(F A) \\
& \operatorname{map}_{T} f=\text { hylo }_{F A}\left(\text { in }_{F B} \circ(F f i d)\right) \text { out }_{F A} \text {, } \\
& \text { where } A=\operatorname{dom}(f) \text { and } B=\operatorname{codom}(f) \\
& \text { cata }_{F} f=\text { hylo }_{F} f \text { out }_{F} \\
& \text { ana }_{F} f=\text { hylo }_{F} \operatorname{in}_{F} f
\end{aligned}
$$

Example 6 (Quicksort). Assuming a type A, and two functions, leq, gt $: A \rightarrow$ List $A \rightarrow$ List $A$, that filter the elements appropriately, we can implement naïve quicksort as:

$$
\begin{array}{lll}
\text { qsort } & : & \text { List } A \rightarrow \text { List } A \\
\text { qsort nil } & = & {[]} \\
\text { qsort }(\operatorname{cons} x l) & = & \text { qsort }(\text { leq } x l)+\text { cons } x(\text { qsort }(\text { gt } x l))
\end{array}
$$

We make the recursive structure explicit by using a tree. The split function unfolds the arguments into this tree, and the join function then flattens it.

$$
\begin{aligned}
& \text { split } \quad: \quad \text { List } A \rightarrow \text { Tree } A \\
& \text { split nil } \quad=\text { empty } \\
& \text { split }(\operatorname{cons} x l)=\operatorname{node}(\operatorname{split}(\text { leq } x l)) x(\operatorname{split}(\text { gt } x l)) \\
& \text { join } \quad: \quad \text { Tree } A \rightarrow \text { List } A \\
& \begin{array}{ll}
\text { join empty } & \text { nil } \\
\text { join }(\text { node } l x r) & =\text { join } l+\text { cons } x(\text { join } r)
\end{array} \\
& \text { qsort : } \quad \text { List } A \rightarrow \text { List } A \\
& \text { qsort }=\text { join } \circ \text { split }
\end{aligned}
$$

We can remove the explicit recursion from these definitions, since split is a tree anamorphism, and join is a tree catamorphism.

$$
\begin{aligned}
& \text { split } \quad: \quad \text { List } A \rightarrow T A(\text { List } A) \\
& \text { split nil } \quad=i n j_{1}() \\
& \text { split }(\text { cons } x l)=\operatorname{inj}_{2}(x \text {, leq } x l \text {, gt } x l) \\
& \text { join } \quad: \quad T A(\text { List } A) \rightarrow \text { List } A \\
& \text { join }\left(i n j_{1}()\right) \quad=\text { nil } \\
& \text { join }\left(i n j_{2}(x, l, r)\right)=l+\text { cons } x r \\
& \text { qsort } \quad: \quad \text { List } A \rightarrow \text { List } A \\
& \text { qsort }=\operatorname{cata}_{T A} \text { join } \circ \operatorname{ana}_{T A} \text { split }
\end{aligned}
$$

Finally, since we have a composition of a catamorphism and an anamorphism, we can write qsort as the equivalent hylomorphism.

$$
\text { qsort }=\text { hylo }_{T A} \text { join split }
$$

The only construct that has not yet been considered is feedback. Although the fixpoint combinator $Y$ can be easily defined as a hylomorphism, we take a different approach. Observe that we can unfold the definition of iter as follows:

$$
\text { iter } f=\mathrm{Y}(\lambda g \cdot(g \nabla i d) \circ f)=(\text { iter } f \nabla i d) \circ f
$$




$$
\begin{array}{ccc}
\rho(f)=A \rightarrow B & \begin{array}{c}
\vdash e_{2}: B \rightarrow C \\
\vdash e_{1}: A \rightarrow B
\end{array} & \vdash e_{1}: F B \rightarrow B \\
\cline { 2 - 3 } & \vdash e_{2} \circ e_{1}: A \rightarrow C \rightarrow F A & \frac{\vdash p: T A \rightarrow T B}{\vdash \operatorname{par}_{T} p: T A \rightarrow T B}
\end{array}
$$

Figure 3: Simple types for Structured Expressions, $E$.

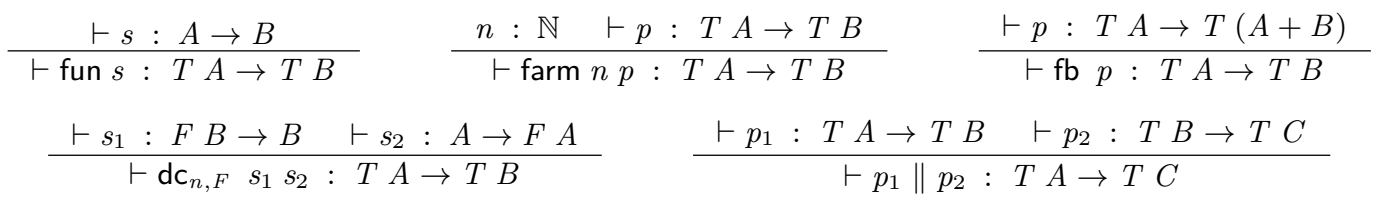

Figure 4: Simple types for Structured Parallel Processes, $P$.

Note that if $f, g: A+B \rightarrow C$, the function $f \nabla g: A+B \rightarrow C$ can be written as the composition of $i d \nabla i d: C+C \rightarrow C$ and $f+g: A+B \rightarrow C+C$. We use this to rewrite iter as follows:

$$
\text { iter } f=(\text { iter } f \nabla i d) \circ f=(i d \nabla i d) \circ(\text { iter } f+i d) \circ f
$$

If $f: A \rightarrow A+B$, we define the functor $(+B)$, with the morphism $(+B) f=f+i d$, which trivially preserves identities and composition. Since iter $f=(i d \nabla i d) \circ(+B)($ iter $f) \circ f$, then:

$$
\text { iter } f=\text { hylo }_{(+B)}(i d \nabla i d) f
$$

\subsection{Structured Expressions}

We have now seen that the denotational semantics of all our parallel constructs can be given in terms of hylomorphisms. This semantic correspondence is not unexpected since it has been used to describe the formal foundations of data-parallel algorithmic skeletons [28]. We take this correspondence one step further by using hylomorphisms as a unifying structure, and by then exploiting the reasoning power provided by the fundamental laws of hylomorphisms. In order to define our type-based approach, we will first define a new language, $E$, that combines two levels, Structured Expressions $(S)$, that enable us to describe a program as a composition of hylomorphisms; and Structured Parallel Processes $(P)$, that build on $S$ using nested algorithmic skeletons. A program in $E$ is then either a structured expression $s \in S$ or a parallel $\operatorname{program}^{\operatorname{par}_{T}} p$, where $p \in P$. Our revised syntax is shown below. Note that since a $p \in P$ can only appear under a par $_{T}$ construct, we no longer need to annotate each fun and dc with the collection $T$ of tasks.

$$
\begin{aligned}
& e \in E \quad:=s \mid \operatorname{par}_{T} p
\end{aligned}
$$

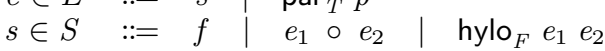

$$
\begin{aligned}
& p \in P \quad:=\text { fun } s\left|p_{1} \| p_{2}\right| \mathrm{dc}_{n, F} s_{1} s_{2} \mid \text { farm } n p \mid \text { fb } p
\end{aligned}
$$

The denotational semantics of $P$ only changes in the rules that mention $e$, and by providing a semantics for $\operatorname{par}_{T}$ :

$$
\begin{aligned}
& \llbracket \operatorname{par}_{T} p \rrbracket \quad=\operatorname{map}_{T} \mathcal{S} \llbracket p \rrbracket \\
& \ddot{\mathcal{S}} \llbracket \text { fun } e \rrbracket \quad=\quad \llbracket e \rrbracket \\
& \mathcal{S} \llbracket \mathrm{dc}_{n, F} e_{1} e_{2} \rrbracket=\text { hylo }_{F} \llbracket e_{2} \rrbracket \llbracket e_{1} \rrbracket
\end{aligned}
$$

The corresponding typing rules are entirely standard (Figures 3-4). Finally, it is convenient to define the "parallelism erasure of $S$ ", $\bar{S}$. Intuitively, $\bar{S}$ contains no nested parallelism: for all $s \in S, s \in \bar{S}$ if and only if $s$ contains no occurrences of the $\operatorname{par}_{T}$ construct. This is equivalent to defining $s$ in the erasure of $S, \bar{S}$, if it is just a composition of atomic functions and hylomorphisms:

$$
s \in \bar{S}::=f \quad \mid \begin{array}{llllll}
s_{1} & \circ & s_{2} & \text { hylo }_{F} & s_{1} & s_{2}
\end{array}
$$

The structure-annotated type system given in Section 4 below describes how to introduce parallelism to an $s \in \bar{S}$ in a sound way.

\subsubsection{Soundness and Completeness.}

It is straightforward to show that the type system from Figs. 3- 4 is both sound and complete wrt our denotational semantics. Our soundness property is: $\forall e \in E ; A, B \in$ Type, $\vdash e: A \rightarrow$ $B \Longrightarrow(\llbracket e \rrbracket \in \llbracket A \rightarrow B \rrbracket)$. The proof is by structural induction over the terms in $E$, using the definitions of $\vdash e: T$ from Figs. $3-$ 4 and $\llbracket . \rrbracket$ above. The corresponding completeness property is: $\forall e \in$ $E ; A, B \in$ Type, $(\llbracket e \rrbracket \in \llbracket A \rightarrow B \rrbracket) \Longrightarrow \vdash e: A \rightarrow B$. The proof is also by structural induction over the terms in $E$, using the

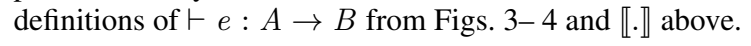

\section{A Type System for Introducing Parallelism}

In this section, we present a rigorous way to introduce parallelism without affecting a program's functional behaviour. We annotate top-level program types with an abstraction of the structure of the program, $\sigma \in \Sigma$. We define the associated type system together with mechanisms for reasoning about these programs using this structure. Intuitively, $\Sigma$ is a "pruned" version of $E$ that retains information about how the computation is performed, while removing as many details as possible about what is being computed.

Definition 4.1 (Families of equivalent programs). We say that an $e \in E$ is in the family of programs that are functionally equivalent to $s \in \bar{S}, e \in E_{s}$, if and only if $e \mathcal{E} s$, for the relation $\mathcal{E}$ that is defined later in this section.

Let $=_{\text {ext }}$ denote extensional equality: $f={ }_{\text {ext }} g \Leftrightarrow \forall x, f x=g x$. Since this is not decidable, we use instead a decidable relation $\mathcal{E}$ which implies extensional equality. Each $E_{s}$ is a family of programs indexed by their structure, i.e. for each family $E_{s}$, there is a function $\phi_{s}: \Sigma \rightarrow E_{s}$ that returns an $e \in E_{s}$ with the desired structure. Note that not all structures $\sigma \in \Sigma$ are indices of a family $E_{s}$, so $\phi_{s}$ is a partial function. Given a structure $\sigma \in \Sigma$ and a $s \in \bar{S}$, we use a superscript, $s^{\sigma}$, as notation for $\phi_{s}(\sigma)$. We define the structure $\Sigma$ and the relation $\mathcal{E}$ later in this section, and the function $\phi_{s}$ in Section 5.

Definition 4.2 (Structure-annotated arrows). Given a structure $\sigma \in$ $\Sigma$, and an $s \in \bar{S}$ with type $A \rightarrow B$, we say that s has type $A \stackrel{\sigma}{\mapsto} B$, if $s$ is equivalent to a parallel program with structure $\sigma$. 


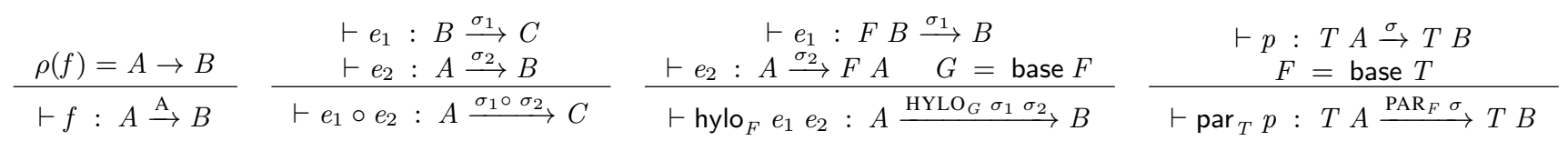

Figure 5: Structure-Annotated Type System for $E$.

\begin{tabular}{|c|c|c|c|c|}
\hline & \multicolumn{2}{|c|}{$\vdash s: A \stackrel{\sigma}{\rightarrow} B$} & \multirow{2}{*}{\multicolumn{2}{|c|}{$\begin{array}{c}\vdash s_{1}: F B \stackrel{\sigma_{1}}{\longrightarrow} B \quad \vdash s_{2}: A \stackrel{\sigma_{2}}{\longrightarrow} F A \quad G=\text { base } F \\
\vdash \mathrm{dc}_{n, F} s_{1} s_{2}: T A \stackrel{\mathrm{DC}_{n, G} \sigma_{1} \sigma_{2}}{\longrightarrow} T B\end{array}$}} \\
\hline & $\vdash$ fun $s: T A$ FUn & $B$ & & \\
\hline$n: \mathbb{N}$ & $\vdash p: T A \stackrel{\sigma}{\rightarrow} T B$ & $\vdash p_{1}$ & $: T A \stackrel{\sigma_{1}}{\longrightarrow} T B \quad \vdash p_{2}: T B \stackrel{\sigma_{2}}{\longrightarrow} T C$ & $\vdash p: T A \stackrel{\sigma}{\rightarrow} T(A+B)$ \\
\hline
\end{tabular}

Figure 6: Structure-Annotated Type System for $P$.

By typechecking $s: A \stackrel{\sigma}{\mapsto} B$, the type system guarantees that there is an equivalent program with structure $\sigma, s^{\sigma} \in E_{s}$. That is, the structured expression $s$ typechecks if and only if $\sigma$ is an index of the family $E_{s}$. The definition of $\phi_{s}$ is actually an algorithm for deriving a parallel program from a sequential program and a typelevel structure, i.e. $\phi_{s}$ provides a mechanism for selecting a parallel program that is equivalent to $s$ and has structure $\sigma$, for well-typed programs. We state this formally in the form of our main soundness and completeness properties later in this section.

\subsection{The Structure-Annotated Type System}

The program structure abstraction, $\sigma \in \Sigma$, is defined below.

$$
\begin{aligned}
& \sigma \in \Sigma \quad::=\sigma_{\mathrm{s}} \mid \operatorname{PAR}_{F} \sigma_{\mathrm{p}} \\
& \sigma_{\mathrm{s}} \in \Sigma_{\mathrm{s}} \quad:=\mathrm{A}|\sigma \circ \sigma| \mathrm{HYLO}_{F} \sigma \sigma \\
& \sigma_{\mathrm{p}} \in \Sigma_{\mathrm{p}} \quad:=\quad \operatorname{FUN} \sigma_{\mathrm{s}} \quad \operatorname{DC}_{n, F} \sigma_{\mathrm{s}} \sigma_{\mathrm{s}} \\
& \left|\sigma_{\mathrm{p}} \| \sigma_{\mathrm{p}}\right| \operatorname{FARM}_{n} \sigma_{\mathrm{p}} \mid \mathrm{FB} \sigma_{\mathrm{p}}
\end{aligned}
$$

Figs. 5- 6 define the annotated type system that extends our simple type system from Figs. 3-4, and that associates expressions $e \in E$ with structures $\sigma \in \Sigma$. As before, the global environment, $\rho$, maps primitive functions to their types. The simple annotated arrow, $e: A \stackrel{\sigma}{\rightarrow} B$, states that $e$ has exactly the structure $\sigma$. In order to define $A \stackrel{\sigma}{\mapsto} B$, we need to extend the type system further with a convertibility relation.

\subsubsection{Convertibility.}

We extend our type system with a non-structural rule that captures the convertibility relation, $\equiv$, for $\Sigma$.

$$
\frac{\vdash e: A \stackrel{\sigma_{1}}{\longrightarrow} B \quad \sigma_{1} \equiv \sigma_{2}}{\vdash e: A \stackrel{\sigma_{2}}{\longmapsto} B}
$$

$\equiv$ is defined in terms of the relations $\equiv_{\mathrm{s}} \in \Sigma_{\mathrm{s}} \times \Sigma_{\mathrm{s}}$ and $\equiv_{\mathrm{p}} \in$ $\Sigma_{\mathrm{p}} \times \Sigma_{\mathrm{p}}$, plus a rule that links the $\Sigma_{\mathrm{s}}$ and $\Sigma_{\mathrm{p}}$ levels, PAR-EQUIV.

$$
\begin{gathered}
\frac{\sigma_{1} \equiv_{\mathrm{s}} \sigma_{2}}{\sigma_{1} \equiv_{2}} \quad \frac{\sigma_{1} \equiv_{\mathrm{p}} \sigma_{2}}{\operatorname{PAR}_{F} \sigma_{1} \equiv \operatorname{PAR}_{F} \sigma_{2}} \\
\operatorname{PAR}_{F}(\text { FUN } \sigma) \equiv \operatorname{MAP}_{F} \sigma \quad \text { (PAR-EQUIV) }
\end{gathered}
$$

The structures MAP and ITER are defined in Section 5, and represent the structures of the corresponding hylomorphisms. We define a number of equivalences, starting with $\equiv_{p}$. A parallel pipeline structure $(\|)$ is functionally equivalent to a function composition; a task farm FARM can be introduced for any structure; and divide-and- conquer DC and feedback FB can be derived from hylomorphisms.

$$
\begin{array}{rllr}
\text { FUN } \sigma_{1} \| \text { FUN } \sigma_{2} & \equiv_{\mathrm{p}} & \text { FUN }\left(\sigma_{2} \circ \sigma_{1}\right) & \text { (PIPE-EQUIV) } \\
\operatorname{DC}_{n}, F \sigma_{1} \sigma_{2} & \equiv_{\mathrm{p}} & \text { FUN }\left(\text { HYLO } F \sigma_{1} \sigma_{2}\right) & \text { (DC-EQUIV) } \\
\text { FARM }_{n} \sigma & \equiv_{\mathrm{p}} & \sigma & (\text { FARM-EQUIV) } \\
\operatorname{FB}(\operatorname{FUN} \sigma) & \equiv_{\mathrm{p}} & \text { FUN }(\text { ITER } \sigma) & (\text { FB-EQUIV) }
\end{array}
$$

These equivalences, plus reflexivity, symmetry and transitivity, define an equational theory that allows conversion between different parallel forms, as well as conversion between structured expressions and parallel forms, as required by our type system. In these equivalences, we implicitly assume the necessary well-formedness constraints: any structure under a FUN or DC must be in $\Sigma_{\mathrm{s}}$, and the structure under FARM must be in $\Sigma_{\mathrm{p}}$. Note that, thanks to the transitivity of $\equiv$, we can use these simple equivalences to derive interesting properties of our parallel structures. For example, the associativity of parallel pipelines does not need to be defined explicitly, since it can be derived from the associativity of composition. We defer the definition of $\equiv_{\mathrm{s}}$ to Section 4.2.

Definition 4.3 (Convertibility in $E$ ). For all convertibility rules in $\Sigma$, there is an equivalent rule in $E$. We define the equivalence relation $\mathcal{E} \in E \times E$ to be the relation $\equiv$ lifted to $E$.

An example that illustrates this is that the PIPE-EQUIV rule corresponds to the rule (fun $s_{1} \|$ fun $\left.s_{2}\right) \quad \mathcal{E}_{\mathrm{p}} \quad\left(\right.$ fun $\left.\left(s_{2} \circ s_{1}\right)\right)$.

Lemma 1. Semantic equivalence. $\forall e_{1}, e_{2} \in E, \quad e_{1} \mathcal{E} e_{2} \Rightarrow$ $\llbracket e_{1} \rrbracket=e_{\text {ext }} \llbracket e_{2} \rrbracket$

Proof Sketch. Straightforward by induction on the structure of the equivalence relation $\mathcal{E}$, using the denotational semantics of $P$, and the laws of hylomorphisms (Section 5).

As a consequence of Lemma 1, the $\mathcal{E}$ relation can be used to define the families $E_{s}$. Any extension to $\equiv$ and $\mathcal{E}$ may expose more opportunities for parallelisation in $E_{s}$. There remains only the definition of the function $\phi_{s}$. We defer this to Section 5, together with the decision procedure for $\equiv$ and $\mathcal{E}$.

\subsubsection{Soundness and Completeness.}

Since the annotated type system is a simple extension of that from Figs 3-4 and since the convertibility rule only applies to structures, it is trivial to show that the new type system is both sound and complete wrt the original system, once structure is removed, since $\forall e \in E, \sigma \in \Sigma, \vdash e: A \stackrel{\sigma}{\mapsto} B \Longrightarrow \vdash e: A \rightarrow B$. We therefore omit these proofs. Our main soundness and completeness 


$$
\begin{aligned}
& \text { hylo }_{F} \text { in }_{F} \text { out }_{F}=i d_{\mu F} \\
& \text { hylo }_{F}(f \circ \eta) g=\text { hylo }_{G} f(\eta \circ g) \Leftarrow \eta: F \rightarrow G \\
& \left(\text { hylo }_{F} f h_{1}\right) \circ\left(\text { hylo }_{F} h_{2} g\right) \quad \Leftarrow h_{1} \circ h_{2}=i d \\
& f_{1} \circ\left(\text { hylo }_{F} g_{1} g_{2}\right) \circ f_{2}=\text { hylo }_{F} g_{1}^{\prime} g_{2}^{\prime} \Leftarrow f_{1} \text { strict } \wedge f_{1} \circ g_{1}=g_{1}^{\prime} \circ F f_{1} \wedge g_{2} \circ f_{2}=F f_{2} \circ g_{2}^{\prime} \\
& \text { hylo }_{F} f g \text { strict }
\end{aligned}
$$

Figure 7: Hylomorphism Laws
HYLO-REFLEX

HYLO-SHIFT

HYLO-COMPOSE

HYLO-FUSION

HYLO-STRICT theorems for convertibility ensure that the type system derives only functionally equivalent parallel structures from structured expressions. The proofs of these properties build on a number of details that are introduced in Section 5.

Theorem 1. Soundness of Conversion.

$$
\forall s \in \bar{S}, \sigma \in \Sigma, \vdash s: A \stackrel{\sigma}{\mapsto} B \Rightarrow s^{\sigma} \in E_{s}
$$

Proof Sketch. Since $s^{\sigma}$ is a synonym for $\phi_{s}(\sigma), s^{\sigma}$ is in $E_{s}$ if $\phi_{s}$ is defined for $\sigma$. This property follows directly from the definition of $\phi_{s}(\sigma)$ (Def. 5.1) and from Thm 3 in Section 5.

A straightforward consequence of the soundness of the conversion and of Lemma 1 is that if a structured expression typechecks with type $A \stackrel{\sigma}{\mapsto} B$, then there always exists a functionally equivalent $e$ whose structure is $\sigma$.

Corollary 1. $\forall s \in \bar{S}, \sigma \in \Sigma, \vdash s: A \stackrel{\sigma}{\longmapsto} B \Rightarrow \exists e \in E$ such that $e: A \stackrel{\sigma}{\rightarrow} B$ and $\llbracket e \rrbracket={ }_{e x t} \llbracket s \rrbracket$.

Theorem 2. Completeness of Conversion.

$$
\forall s \in \bar{S} ; \sigma, \sigma^{\prime} \in \Sigma ; s: A \stackrel{\sigma^{\prime}}{\longrightarrow} B \wedge s^{\sigma} \in E_{s} \Rightarrow \vdash s: A \stackrel{\sigma}{\mapsto} B
$$

Proof Sketch. This follows directly from the definition of $\phi_{s}(\sigma)$ (Def. 5.1) and Thm 3 in Sec. 5.

\subsection{Functional Equivalence}

The proofs of soundness and completeness rely on a decision procedure for $\equiv$, as well as on the definition of the $\phi_{s}$ function for the families $E_{s}$. The definition of $\equiv$ requires a definition of $\equiv_{s} \in \Sigma_{\mathrm{s}} \times$ $\Sigma_{\mathrm{s}}$. Our $\equiv_{\mathrm{s}}$ adapts the well known hylomorphism laws (Fig. 7) [5, $10,22]$, using restricted instances of those laws. These restrictions serve two purposes: i) we avoid checking strictness conditions by simply ensuring that all the functions we use are strict; ii) because the equivalences that we can capture are very limited if we assume no knowledge of atomic functions, due to the side conditions on the rules, we expose extra structure in our programs.

1. We explicitly represent the $\mathrm{in}_{F}$, out $_{F}$ and id functions, with the obvious denotational semantics.

2. We explicitly represent the section of a bifunctor $F$ applied to a structured expression $s$, as $F s$ rather than $F s$ id. This, plus the strictness assumption, enables us to apply some limited forms of HYLO-SHIFT and HYLO-FUSION.

3. We explicitly represent $\Delta$ and $\nabla$ (Fig. 1). Although we do not define equivalences for these combinators, we use them to define the ITER structure later.

$$
\begin{array}{lll}
s \in S & := & f \mid\left\langle\text { prim }_{\mid}\left|e_{1} \circ e_{2}\right| \text { hylo }_{F} e_{1} e_{2}\right. \\
\text { prim } & := & \text { in }_{F} \mid \text { out }_{F} \mid \text { id }\left|e_{1}\langle o p\rangle e_{2}\right| F e \\
\text { op } & :== & \nabla \mid \triangle \\
\sigma_{\mathrm{s}} \in \Sigma_{\mathrm{s}} & ::= & \ldots \mid \text { IN } \mid \text { oUT } \mid \text { ID }\left|\sigma_{1}\langle o p\rangle \sigma_{2}\right| F \sigma
\end{array}
$$

With these structures, we can define the special cases of $\mathrm{HYLO}_{F}$ :

$$
\begin{array}{ll}
\operatorname{MAP}_{F}, \operatorname{CATA}_{F}, \text { ANA }_{F} & : \Sigma \rightarrow \Sigma \\
\operatorname{MAP}_{F} \sigma & =\operatorname{HYLO}_{F}(\text { IN } \circ F) \text { OUT } \\
\operatorname{CATA}_{F} \sigma & =\mathrm{HYLO}_{F} \sigma \text { OUT } \\
\operatorname{ANA}_{F} \sigma & =\mathrm{HYLO}_{F} \text { IN } \sigma \\
\operatorname{ITER} \sigma & =\mathrm{HLO}_{(+)}(\mathrm{ID} \nabla \text { ID }) \sigma
\end{array}
$$

The typing rules are then easily extended.

$$
\begin{aligned}
& \vdash \mathrm{id}: A \stackrel { \mathrm { ID } } { \longrightarrow } A \quad \longdiv { \vdash \operatorname { i n } _ { F } : F ( \mu F ) \stackrel { \mathrm { IN } } { \longrightarrow } \mu F } \\
& \vdash \text { out }_{F}: \mu F \stackrel{\text { OUT }}{\longrightarrow} F(\mu F) \\
& \frac{\vdash e: A \stackrel{\sigma}{\rightarrow} B}{\vdash F e: F A C \stackrel{F \sigma}{\longrightarrow} F B C} \\
& \frac{\vdash e_{1}: A \stackrel{\sigma_{1}}{\longrightarrow} B \quad \vdash e_{2}: A \stackrel{\sigma_{2}}{\longrightarrow} C}{\vdash e_{1} \Delta e_{2}: A \stackrel{\sigma_{1} \Delta \sigma_{2}}{\longrightarrow} B \times C} \\
& \frac{\vdash e_{1}: A \stackrel{\sigma_{1}}{\longrightarrow} C \vdash e_{2}: \quad \vdash e_{2}: B \stackrel{\sigma_{2}}{\longrightarrow} C}{\vdash e_{1} \nabla e_{2}: A+B \stackrel{\sigma_{1} \nabla \sigma_{2}}{\longrightarrow} C}
\end{aligned}
$$

The convertibility relation is also extended to include some equivalences that are derived from the hylomorphism laws:

$\begin{array}{cccc}\text { ID } \circ \sigma & \equiv_{\mathrm{s}} & \sigma & \text { (ID-LEFT) } \\ \sigma \circ \text { ID } & \equiv_{\mathrm{s}} & \sigma & \text { (ID-RIGHT) } \\ \text { OUT } \circ \text { IN } & \equiv_{\mathrm{s}} & \text { ID } & \text { (OUT-IN-ID) } \\ \text { IN } \circ \text { OUT } & \equiv_{\mathrm{s}} & \text { ID } & \text { (IN-OUT-ID) } \\ \mathrm{HYLO}_{F} \text { IN OUT } & \equiv_{\mathrm{s}} & \text { ID } & \text { (HYLO-ID) } \\ F\left(\sigma_{1} \circ \sigma_{2}\right) & \equiv_{\mathrm{s}} & F \sigma_{1} \circ F \sigma_{2} & \text { (F-COMP) } \\ \mathrm{HYLO}_{F} \sigma_{1} \sigma_{2} & \equiv_{\mathrm{s}} & \mathrm{CATA}_{F} \sigma_{1} \circ \mathrm{ANA}_{F} \sigma_{2} & \text { (HYLO-COMP) } \\ \mathrm{CATA}_{F}\left(\sigma_{1} \circ F \sigma_{2}\right) & \equiv_{\mathrm{s}} & \mathrm{CATA}_{F} \sigma_{1} \circ \mathrm{MAP}_{F} \sigma_{2} & \text { (CATA-COMP) } \\ \text { ANA } F_{F}\left(F \sigma_{1} \circ \sigma_{2}\right) & \equiv_{\mathrm{s}} & \mathrm{MAP}_{F} \sigma_{1} \circ \mathrm{ANA}_{F} \sigma_{2} & \text { (ANA-COMP) } \\ \text { ANA } F_{F}\left(F \sigma_{1} \circ \text { OUT }\right) & \equiv_{\mathrm{s}} & \mathrm{MAP}_{F} \sigma_{1} & \text { (ANA-MAP) }\end{array}$

We extend $\mathcal{E}$ in the expected way with the lifted $\equiv_{s}, \mathcal{E}_{\mathrm{s}}$. The rule HYLO-COMP is derived from the HyLO-Compose law. The rules CATA-COMP and ANA-COMP are derived from HYLO-FUSION. It is easy to see how any strictness condition holds in those rules. Finally, the rule ANA-MAP is derived from the HYLO-SHIFT law. It is used only to give a uniform representation of the $\mathrm{MAP}_{F}$ structure.

\section{Determining Functional Equivalence}

Recall that for all $s \in \bar{S}$, there is a $\Sigma$-indexed family $E_{s}$. For all well-typed structured expression $s: A \stackrel{\sigma}{\longmapsto} B, \sigma$ is an index of the family defined by $s$, i.e. $s^{\sigma} \in E_{s}$. The function $\phi_{s}: \Sigma \rightarrow E_{s}$ is a partial function whose result is defined for any structure $\sigma$ that is an index of the family $E_{s}$. Given an $s: A \stackrel{\sigma^{\prime}}{\rightarrow} B$, both the typechecking algorithm and the function $\phi_{s}$ need to decide whether $\sigma \equiv \sigma^{\prime}$. This problem has been extensively studied for bicartesian closed categories $[7,13,35]$, and it is beyond the scope of this paper 


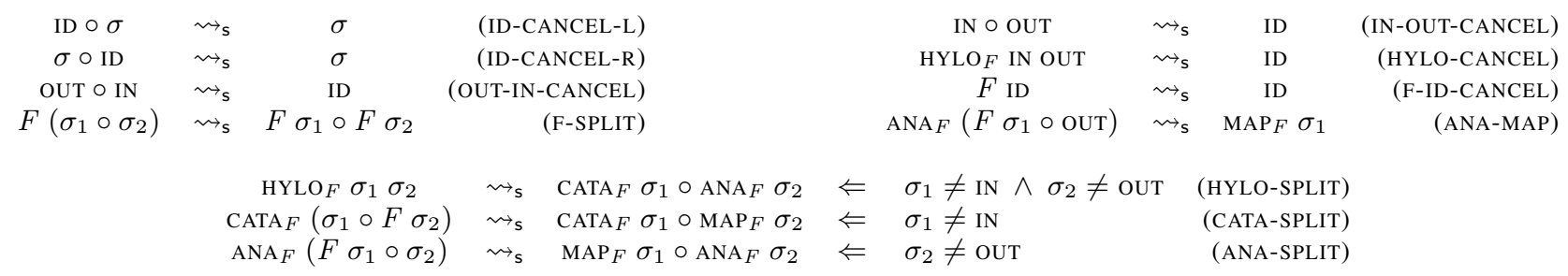

Figure 8: Rewriting system in $S$

to produce a novel decision procedure for the equality of terms. We consequently use a basic decision procedure, but one that enables interesting parallelisations.

\subsection{Reforestation.}

We take the standard approach of using term rewriting systems to decide equality in $\Sigma$ (and hence $E$ ). It is well known that if a rewriting system is confluent, then two terms have the same normal form if and only if they are equal with respect to the underlying equational theory. If we define a confluent term rewriting system with $\equiv$ as underlying theory, we can use the syntactic equality of normalised forms as our decision procedure. We present the rewriting system in two parts. The first part is derived from orienting the rules in $\equiv$ so that parallelism is erased:

$\begin{array}{lll}\text { FARM }_{n} \sigma_{\mathrm{p}} & \rightsquigarrow \mathrm{p} & \sigma_{\mathrm{p}} \\ \text { FUN } \sigma_{1} \| \text { FUN } \sigma_{2} & \rightsquigarrow_{\mathrm{p}} & \text { FUN }\left(\sigma_{1} \circ \sigma_{2}\right) \\ \text { DC }_{n, F} \sigma_{1} \sigma_{2} & \rightsquigarrow_{\mathrm{p}} & \text { FUN }\left(\text { HYLO } F \sigma_{1} \sigma_{2}\right) \\ \text { FB }\left(\text { FUN } \sigma_{1}\right) & \rightsquigarrow_{\mathrm{p}} & \text { FUN }\left(\text { ITER } \sigma_{1}\right) \\ \text { PAR }_{T}\left(\text { FUN } \sigma_{\mathrm{s}}\right) & \rightsquigarrow_{\mathrm{p}} & \text { MAP }_{T} \sigma_{\mathrm{s}}\end{array}$

The first four rules rewrite terms in $\Sigma_{\mathrm{p}}$ to terms in $\Sigma_{\mathrm{p}}$, and the last rule rewrites terms in $\Sigma$ to terms in $\Sigma$. We define $\overline{\Sigma_{s}}$ in an analogous way to $\bar{S}$, and erase as any normalisation procedure for a rewriting system $\rightsquigarrow_{\mathrm{p}}$ :

$$
\begin{array}{lll}
\text { erase } & : \quad \Sigma \rightarrow \bar{\Sigma}_{\mathrm{s}} \\
\text { erase } \sigma & = & \sigma^{\prime}, \text { s.t. } \sigma \rightsquigarrow_{\mathrm{p}}^{*} \sigma^{\prime} \wedge \nexists \sigma^{\prime \prime} \text { s.t. } \sigma^{\prime \prime} \rightsquigarrow_{\mathrm{p}} \sigma^{\prime \prime}
\end{array}
$$

Lemma 2. The rewriting system $\rightsquigarrow_{\mathrm{p}}$ is confluent.

Proof Sketch. The rewriting system is terminating, since the number of redexes is precisely the number of parallel structures (including $\operatorname{PAR}_{T}$ ), which is reduced following each rewriting step. It is also easy to show that any critical pairs arising from these rules have the same normal form. For example, a farm of a pipeline reduces to the same expression regardless of which structure is erased first. By Newman's lemma [1] we can conclude that $\rightsquigarrow_{p}$ is confluent.

Since $\rightsquigarrow_{p}$ is confluent, we know that the result of erase is unique. Recall that all the results that are derived from the equational theory $\equiv$ can be lifted to $\mathcal{E}$. This implies that there is an erase $E$ : $E \rightarrow \bar{S}$ procedure that is equivalent to erase defined with the rewritings lifted to $E$. The second step is the normalisation of $\sigma \in$ $\bar{\Sigma}_{\mathrm{s}}$. We once again use a confluent rewriting system derived from $\equiv_{\mathrm{s}}$, and define it modulo associativity of the composition $\circ$. The direction of the rewriting is chosen so a "reforestation" rewriting is performed. Hylomorphisms are first split into catamorphisms and anamorphisms, which are then themselves split into compositions of maps, catamorphisms and anamorphisms. We omit some trivial cases, e.g. $F \sigma \circ F \sigma^{-1} \rightsquigarrow$ ID, and prioritise the rules that deal with ID to simplify the confluence of the rewriting system. We only consider the inverses IN and out, $F$ IN and $F$ ouT, etc. For uniformity reasons, ANA-MAP is applied to the anamorphisms that perform a map computation.

Lemma 3. The term rewriting system $\rightsquigarrow_{\mathrm{s}}$ is confluent.

Proof Sketch. The rewriting system is terminating, since the preconditions of the rules ensure that no cycles are introduced. The rewriting system is also locally confluent. It is trivial to observe that any critical pair arising from the ID rules have the same normal form. The critical pairs arising from rules MAP-SPLIT and CATA/ANA-SPLIT can be reduced to the same normal form, by applying F-SPLIT and CATA/ANA-SPLIT and/or ANA-MAP in a different order. For example, we can rewrite any $\operatorname{CATA}_{F}\left(\sigma_{1} \circ F\left(\sigma_{2} \circ \sigma_{3}\right)\right) \rightsquigarrow_{s}^{*}$ $\operatorname{CATA}_{F} \sigma_{1} \circ \operatorname{MAP}_{F} \sigma_{2} \circ \mathrm{MAP}_{F} \sigma_{3}$. Any problems that appear from the critical pairs of the ID rules and the SPLIT rules can be solved by forcing the ID rules to be applied first, and working modulo associativity. As before, Newman's lemma completes the proof.

Finally, we define the normalisation procedures for $\bar{\Sigma}_{\mathrm{s}}$ and $\Sigma$.

$$
\begin{array}{ll}
\operatorname{norm}_{\mathrm{s}} \sigma & =\sigma^{\prime}, \text { s.t. } \sigma \rightsquigarrow_{\mathrm{s}}^{*} \sigma^{\prime} \wedge \nexists \sigma^{\prime \prime} \text { s.t. } \sigma^{\prime} \rightsquigarrow_{\mathrm{s}} \sigma^{\prime \prime} \\
\text { norm } & =\text { norm }
\end{array}
$$

We use a subscript, norm ${ }_{E}$, to denote this normalisation procedure lifted to $E$. Given that the underlying equational theory of the term rewriting system is $\mathcal{E}$, we know that: $\forall e_{1}, e_{2} \in E,\left(\right.$ norm $_{E} e_{1}=$ $\left.\operatorname{norm}_{E} e_{2}\right) \Leftrightarrow\left(e_{1} \leftrightarrow{ }^{*} e_{2}\right) \Leftrightarrow\left(e_{1} \mathcal{E} e_{2}\right)$.

Theorem 3 (norm defines a decision procedure for $\equiv$ ). For all $\sigma_{1}, \sigma_{2} \in \Sigma, \sigma_{1} \equiv \sigma_{2}$ if and only if norm $\sigma_{1}=$ norm $\sigma_{2}$.

Proof Sketch. From the properties of $\rightsquigarrow_{\mathrm{p}}$, we derive that it is always true that $\sigma_{i} \equiv$ erase $\sigma_{i}$. Since $\rightsquigarrow_{\mathrm{s}}$ is confluent, by the properties of term rewriting systems, we know that erase $\sigma_{1} \equiv$ erase $\sigma_{2}$ if and only if norms $\left(\operatorname{erase}\left(\sigma_{1}\right)\right)=\operatorname{norm}_{\mathbf{s}}\left(\operatorname{erase}\left(\sigma_{2}\right)\right)$. We finish the proof by combining these two facts using the transitivity of $\equiv$ with the definition of norm.

The fact that we can lift the results from $\Sigma$ to $E$ implies that we can use this rewriting system not only to reason about program equivalences, but also to define an algorithm to derive a parallel program from some $s \in \bar{S}$ and a type-level parallel structure. We sketch this algorithm as the definition of $\phi_{s}$.

Definition $5.1\left(\phi_{s}\right)$. Let $s \in \bar{S}, \sigma_{1} \in \bar{\Sigma}_{\mathrm{s}}$, such that $\vdash s: A \stackrel{\sigma_{1}}{\longrightarrow} B$, and $\sigma_{2} \in \Sigma$. We define $\phi_{s}\left(\sigma_{2}\right)$ as follows:

Let $\sigma_{i}^{\prime}=\operatorname{norm} \sigma_{i}$. If $\sigma_{1}^{\prime}=\sigma_{2}^{\prime}$, then:

1. Reverse the rewriting steps from $\sigma_{2}$ to $\sigma_{2}^{\prime}: \sigma_{2}^{\prime} \rightsquigarrow{ }^{*} \sigma_{2}$.

2. Obtain the proof of $\sigma_{1} \equiv \sigma_{2}$ by using $\sigma_{1} \rightsquigarrow * \sigma_{1}^{\prime}$ and (1).

3. Obtain the rewriting steps $\sigma_{1} \rightsquigarrow{ }^{*} \sigma_{2}$ from (2).

4. Lift the rewriting steps to $E$, and apply them to $s: s{ }_{E}^{*} e$. 


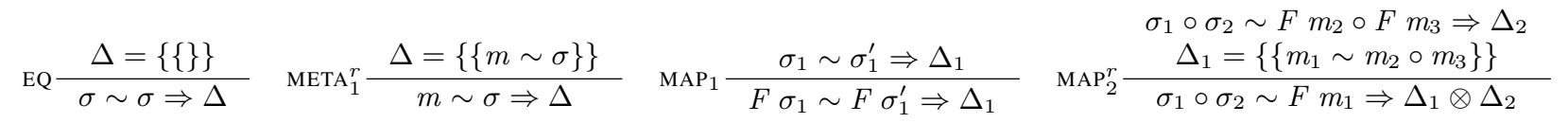

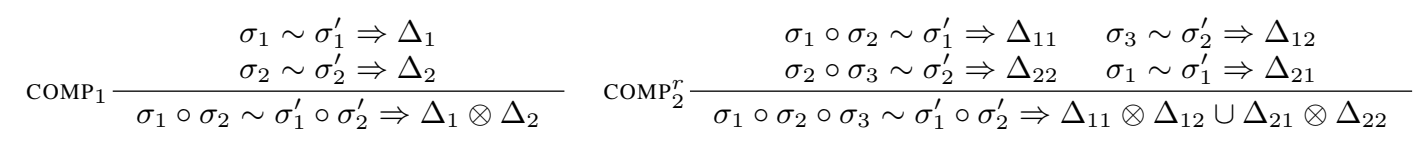

$$
\begin{aligned}
& \mathrm{OP} \frac{\sigma_{1} \sim \sigma_{1}^{\prime} \Rightarrow \Delta_{1} \quad \sigma_{2} \sim \sigma_{2}^{\prime} \Rightarrow \Delta_{2}}{\sigma_{1}\langle o p\rangle \sigma_{2} \sim \sigma_{1}^{\prime}\langle o p\rangle \sigma_{2}^{\prime} \Rightarrow \Delta_{1} \otimes \Delta_{2}} \quad \mathrm{HYLO}_{1} \frac{\sigma_{1} \sim \sigma_{1}^{\prime} \Rightarrow \Delta_{1} \quad \sigma_{2} \sim \sigma_{2}^{\prime} \Rightarrow \Delta_{2}}{\mathrm{HYLO}_{F} \sigma_{1} \sigma_{2} \sim \mathrm{HYLO}_{F} \sigma_{1}^{\prime} \sigma_{2}^{\prime} \Rightarrow \Delta_{1} \otimes \Delta_{2}} \\
& \mathrm{HYLO}_{2}^{r} \frac{\sigma_{1} \circ \sigma_{2} \sim \mathrm{HYLO}_{F} m_{1} \text { OUT } \circ \mathrm{HYLO}_{F} \text { IN } m_{2} \Rightarrow \Delta_{1} \quad \sigma_{1} \circ \sigma_{2} \sim \mathrm{HYLO}_{F} m_{1} \text { OUT } \Rightarrow \Delta_{2} \sigma_{1} \circ \sigma_{2} \sim \mathrm{HYLO}_{F} \text { IN } m_{2} \Rightarrow \Delta_{3}}{\sigma_{1} \circ \sigma_{2} \sim \mathrm{HYLO}_{F} m_{1} m_{2} \Rightarrow \Delta_{1} \cup\left\{\left\{m_{2} \sim \text { OUT }\right\}\right\} \otimes \Delta_{2} \cup\left\{\left\{m_{1} \sim \mathrm{IN}\right\}\right\} \otimes \Delta_{3}} \\
& \sigma_{1} \circ \sigma_{2} \sim \mathrm{HYLO}_{F}\left(\mathrm{IN} \circ F m_{2}\right) \text { OUT } \circ \mathrm{HYLO}_{F} \text { IN } m_{3} \Rightarrow \Delta_{1} \\
& \begin{array}{c}
\sigma_{1} \circ \sigma_{2} \sim \mathrm{HYLO}_{F}\left(\mathrm{IN} \circ F m_{2}\right) \text { OUT } \circ \mathrm{HYLO}_{F} \text { IN } m_{3} \Rightarrow \Delta_{1} \\
\mathrm{HYLO}_{3}^{r} \frac{\sigma_{1} \circ \sigma_{2} \sim \mathrm{HYLO}_{F}\left(\mathrm{IN} \circ F m_{2}\right) \text { OUT } \Rightarrow \Delta_{2} \quad \Delta=\left\{\left\{m_{1} \sim F m_{2} \circ m_{3}\right\}\right\}}{\sigma_{1} \circ \sigma_{2} \sim \mathrm{HYLO}_{F} \text { IN } m_{1} \Rightarrow \Delta \otimes \Delta_{1} \cup \Delta \otimes\left\{\left\{m_{3} \sim \text { OUT }\right\}\right\} \otimes \Delta_{2}}
\end{array} \\
& \mathrm{HYLO}_{4}^{r} \frac{\sigma_{1} \circ \sigma_{2} \sim \mathrm{HYLO}_{F} m_{2} \text { OUT } \circ \mathrm{HYLO}_{F}\left(\mathrm{IN} \circ F m_{3}\right) \text { OUT } \Rightarrow \Delta}{\sigma_{1} \circ \sigma_{2} \sim \mathrm{HYLO}_{F} m_{1} \text { OUT } \Rightarrow\left\{\left\{m_{1} \sim m_{2} \circ F m_{3}\right\}\right\} \otimes \Delta} \\
& \operatorname{HYLO}_{5}^{r} \frac{\sigma_{1} \circ \sigma_{2} \sim \mathrm{HYLO}_{F} m_{1} \text { OUT } \circ \mathrm{HYLO}_{F} \text { IN } \sigma_{3} \Rightarrow \Delta_{1} \quad \sigma_{1} \circ \sigma_{2} \sim \operatorname{norm}\left(\mathrm{HYLO}_{F} \text { IN } \sigma_{3}\right) \Rightarrow \Delta_{2} \quad \sigma_{3} \neq \text { OUT }}{\sigma_{1} \circ \sigma_{2} \sim \mathrm{HYLO}_{F} m_{1} \sigma_{3} \Rightarrow \Delta_{1} \cup\left\{\left\{m_{1} \sim \operatorname{IN}\right\}\right\} \otimes \Delta_{2}} \\
& \operatorname{HYLO}_{6}^{r} \frac{\sigma_{1} \circ \sigma_{2} \sim \mathrm{HYLO}_{F} \sigma_{3} \text { OUT } \circ \mathrm{HYLO}_{F} \text { IN } m_{1} \Rightarrow \Delta_{1} \quad \sigma_{1} \circ \sigma_{2} \sim \operatorname{norm}\left(\mathrm{HYLO}_{F} \sigma_{3} \text { OUT }\right) \Rightarrow \Delta_{2} \quad \sigma_{3} \neq \mathrm{IN}}{\sigma_{1} \circ \sigma_{2} \sim \mathrm{HYLO}_{F} \sigma_{3} m_{1} \Rightarrow \Delta_{1} \cup\left\{\left\{m_{1} \sim \text { OUT }\right\}\right\} \otimes \Delta_{2}} \\
& \mathrm{HYLO}_{7}^{r} \frac{\sigma_{1} \circ \sigma_{2} \sim \mathrm{HYLO}_{F}\left(\mathrm{IN} \circ F m_{2}\right) \text { OUT } \circ \mathrm{HYLO}_{F}\left(\mathrm{IN} \circ F m_{3}\right) \text { OUT } \Rightarrow \Delta}{\sigma_{1} \circ \sigma_{2} \sim \mathrm{HYLO}_{F}\left(\mathrm{IN} \circ F m_{1}\right) \text { OUT } \Rightarrow\left\{\left\{m_{1} \sim m_{2} \circ m_{3}\right\}\right\} \otimes \Delta}
\end{aligned}
$$

Figure 9: Unification Rules.

Since the typechecking algorithm for our type system needs to decide $\sigma_{1} \equiv \sigma_{2}$ (e.g. using Thm. 3), steps (1) to (2) can be omitted if we know that $\vdash s: A \stackrel{\sigma_{2}}{\longmapsto} B$ (recall the proof of Thm. 1). Conversely, if there is some $s \vdash A \stackrel{\sigma_{1}}{\longrightarrow} B$, and $s^{\sigma_{2}} \in E_{s}$, we know that there is a proof $\sigma_{1} \equiv \sigma_{2}$ (step (2) in Def. 5.1), and therefore $s \vdash A \stackrel{\sigma_{2}}{\longmapsto} B$ (recall the proof of Thm. 2).

\subsection{Structure Unification.}

The structure-annotated types that we have presented so far require the specification of a full structure $\sigma \in \Sigma$. However, it is sometimes sufficient, or desirable, to specify only the relevant parts of this structure. We allow this by introducing structure metavariables in $\Sigma$. Selecting suitable substitutions for these metavariables can be automated in different ways, as we will see later in this section. Given a set of metavariables, $\mathcal{M}$, we extend the syntax of $\Sigma$ as follows:

$$
\begin{aligned}
& m \in \mathcal{M} \quad \sigma \in \Sigma \quad::=\ldots \mid m \\
& \sigma_{\mathrm{s}} \in \Sigma_{\mathrm{s}}::=\ldots\left|m \quad \sigma_{\mathrm{p}} \in \Sigma_{\mathrm{p}}::=\ldots\right| m
\end{aligned}
$$

The underscore character denotes a fresh metavariable, e.g. given a fresh metavariable $m, \operatorname{FARM}_{n}$ - is equivalent to $\operatorname{FARM}_{n} m$.

Definition 5.2 (Substitution Environments). A substitution environment $\delta$ is a mapping of metavariables to structures, $\left\{m_{1} \sim\right.$ $\left.\sigma_{1}, m_{2} \sim \sigma_{2}, \ldots\right\}$. We use $\Delta$ to denote sets of environments $\delta$.

The two basic operations with substitution environments are the application and the extension. We apply a substitution environment $\delta$ to a structure $\sigma$, denoted by $\delta \sigma$, by replacing all metavariables as defined by $\delta$. The extension of $\delta_{1}$ with $\delta_{2}, \delta_{1} \delta_{2}$, is defined in the expected way. If both substitution environments introduce a cycle or conflicting metavariables, the operation fails. Finally, for sets of substitution environments, we define the set of extensions:

$$
\Delta_{1} \otimes \Delta_{2}=\left\{\delta_{1} \delta_{2} \mid \delta_{1} \in \Delta_{1} \wedge \delta_{2} \in \Delta_{2}\right\}
$$

Lemma 4. For all substitutions $\delta$, for all $\sigma \in \Sigma$, norm $\delta \sigma \equiv$ $\delta(\operatorname{norm} \sigma)$.

Proof Sketch. It is obvious that if $\sigma_{1} \equiv \sigma_{2}$, then $\delta \sigma_{1} \equiv \delta \sigma_{2}$, since $\delta$ will apply the same substitution in both $\sigma_{1}$ and $\sigma_{2}$. Since $\sigma \equiv$ norm $\sigma$, we conclude using the reflexivity of $\equiv$.

Note that we can no longer use the relation $\equiv$ in our typechecking rules, since it does not handle metavariables. Instead, we define the relation $\cong$, and define a decision procedure for it.

Definition 5.3 (Equivalence of the Unified Forms). We say that $\sigma_{1} \cong \sigma_{2}$ if there is at least a substitution $\delta$ that makes $\delta \sigma_{1} \equiv \delta \sigma_{2}$.

$$
\sigma_{1} \cong \sigma_{2} \doteq \exists \delta, \delta \sigma_{1} \equiv \delta \sigma_{2}
$$

The type rule for equivalence changes to use the new relation:

$$
\frac{\vdash e: A \stackrel{\sigma_{1}}{\longrightarrow} B \quad \sigma_{1} \cong \sigma_{2}}{\vdash e: A \stackrel{\sigma_{2}}{\longmapsto} B}
$$

In order to typecheck a structured expression with a structure containing metavariables, we need to: i) modify the normalisation procedure; and ii) define a unification algorithm. The normalisation procedure is modified as follows: 
1. Any erase step on a structure with meta-variables always succeeds by simply adding new meta-variables and a substitution environment $\delta$ for those metavariables, e.g.

$$
\begin{aligned}
& m_{1} \| m_{2} \rightsquigarrow \text { FUN }\left(m_{2}^{\prime} \circ m_{1}^{\prime}\right) \\
& \quad \delta=\left\{m_{1} \sim \text { FUN } m_{1}^{\prime}, m_{2} \sim \text { FUN } m_{2}^{\prime}\right\} .
\end{aligned}
$$

2. The constraints of the rules used by the norm ${ }_{S}$ procedure are modified so that they are never satisfied by metavariables, e.g.

$$
\begin{aligned}
& \mathrm{HYLO}_{F} \sigma_{1} \sigma_{2} \rightsquigarrow_{\mathrm{s}} \mathrm{CATA}_{F} \sigma_{1} \circ \mathrm{ANA}_{F} \sigma_{2} \quad \text { (HYLO-SPLIT) } \\
& \Leftarrow \sigma_{1} \neq \mathrm{IN} \wedge \sigma_{2} \neq \text { OUT } \wedge \sigma_{1} \notin \mathcal{M} \wedge \sigma_{2} \notin \mathcal{M}
\end{aligned}
$$

Although condition 2 is not necessary, it simplifies the unification of structures where $\sigma_{1}$ or $\sigma_{2}$ can be unified to IN or OUT.

Unification Rules. Since unifying two structures may lead to different, but valid unifying substitutions, the unification rules yield the set of all possible unifying substitutions, $\Delta$. Disambiguating such situations can be done using cost models, or some other procedure. The rules for unification (Fig. 9) define a unification modulo associativity (rule $\mathrm{COMP}_{2}$ ). Each rule with superscript $r$ has a symmetric version $l$. A statement $\sigma_{1} \sim \sigma_{2} \Rightarrow \Delta$ means that structure $\sigma_{1}$ unifies with structure $\sigma_{2}$, under a non-empty set of substitutions, $\Delta \neq \varnothing$. Whenever two structures do not correspond to the same syntactic structure, the unification rules make any valid assumption about the metavariables that would allow further rewritings to take place.

Theorem 4 (Soundness of the Unification). For all $\sigma_{1}, \sigma_{2} \in \Sigma$,

$$
\sigma_{1} \sim \sigma_{2} \Rightarrow \Delta \Longrightarrow \Delta \neq \varnothing \wedge \forall \delta \in \Delta, \delta \sigma_{1} \equiv \delta \sigma_{2}
$$

Theorem 5 (Completeness of the Unification). For all $\sigma_{1}, \sigma_{2} \in \Sigma$, and substitution $\delta$,

$$
\delta \sigma_{1} \equiv \delta \sigma_{2} \Longrightarrow \exists \Delta \text { s.t. } \Delta \neq \varnothing \wedge \sigma_{1} \sim \sigma_{2} \Rightarrow \Delta
$$

The proofs of those theorems are standard proofs by induction on the derivations of $\sim$ and $\equiv$, and by case analysis on the metavariables.

Corollary 2. $\cong: \sigma_{1} \cong \sigma_{2} \Leftrightarrow$ norm $\sigma_{1} \sim$ norm $\sigma_{2} \Rightarrow \Delta$, i.e. the unification algorithm can be used as a decision procedure for $\cong$.

Proof Sketch. For the proof of the $\Rightarrow$ case, we know that there is at least a $\delta$ such that $\delta \sigma_{1} \equiv \delta \sigma_{2}$. From the properties of $\equiv$, we know that norm $\delta \sigma_{1}=$ norm $\delta \sigma_{2}$. Using Lemma 4, we derive that $\delta\left(\right.$ norm $\left.\sigma_{1}\right) \equiv \delta\left(\right.$ norm $\left.\sigma_{2}\right)$. The completeness of the unification allows us to conclude that norm $\sigma_{1} \sim$ norm $\sigma_{2} \Rightarrow \Delta$.

The proof of $\Leftarrow$ follows from the soundness of the unification algorithm. We know that norm $\sigma_{1} \sim$ norm $\sigma_{2} \Rightarrow \Delta$ implies that $\Delta$ is non-empty, and that for all $\delta \in \Delta, \delta$ (norm $\left.\sigma_{1}\right) \equiv$ $\delta$ (norm $\sigma_{2}$ ). We conclude by selecting any $\delta$ from $\Delta$, and then using Lemma 4.

Using metavariables in structures has some implications. Given a $s \vdash A \stackrel{\sigma_{1}}{\longrightarrow} B$, and a structure $\sigma_{2}$ containing one or more metavariables, $\sigma_{2}$ can no longer be used as an index for a family $E_{s}$. Since there may be alternative, but valid substitutions for the metavariables, it follows that $s^{\sigma_{2}} \subseteq E_{s}$. Given a unification $\sigma_{1} \sim$ $\sigma_{2} \Rightarrow \Delta$, we need to apply a $\bar{\delta} \in \Delta$ to $\sigma_{2}$ in order to use it as an index, $s^{\delta \sigma_{2}} \in E_{s}$. This implies that there are many ways to use our approach. On one hand, fixing this $\sigma_{2}$ to be a closed structure without any metavariables, or one that unifies with $\sigma_{1}$ with a unique substitution, provides a way to manually parallelise a program. On the other hand, if $\sigma_{2}$ is defined to be a metavariable, then a fully automated method for selecting a parallel structure would be needed. In between, there are a wide range of semi-automated possibilities that can be used to reason about the introduction of parallelism to a program. The automated selection mechanism for a $\delta \in \Delta$ can easily be extended with further parallelisation opportunities. Furthermore, it can be parameterised by architecture-specific details, so that compiling a program for different architectures leads to alternative parallelisations. This is, however, beyond the scope of this paper.

Compositionality and Higher-Order Structured-Arrows. We finish this section with a discussion of the compositionality of our approach. Most of this paper deals with the typing rule for structure-annotated arrows. Extending our work for a language with definitions and with a limited-form of higher-order structured arrows can be done using the unification rules from Fig. 9. Applying some $e: A \stackrel{\sigma_{1}^{\prime}}{\longmapsto} B$ to a $f: A \stackrel{\sigma_{1}}{\longmapsto} B \rightarrow C \stackrel{\sigma_{2}}{\longmapsto} D$ typechecks only if $\sigma_{1} \sim \sigma_{1}^{\prime} \Rightarrow \Delta$, and the type of this would be annotated with a structure resulting from applying any $\delta \in \Delta$, $f e: C \stackrel{\delta \sigma_{2}}{\longmapsto} D$. One advantage of this is to easily allow the specification of new structures that can be later used in our programs. The corresponding erasure rules would then be derived automatically from such a specification. We would need, however, to verify a back-end for such a language in order to ensure that the operational semantics of the new structures are sound with respect to the specification. Although we do not explain this idea in detail in this paper, we will use a small example of a defined structure in Section 6.3.

Although it seems entirely feasible to support full higher-order structured arrows, there is the question of whether this is desirable: what would types such as $A \stackrel{\sigma}{\longmapsto} B \stackrel{\sigma^{\prime}}{\longmapsto} C$ or $(A \stackrel{\sigma}{\longmapsto} B) \stackrel{\sigma^{\prime}}{\longmapsto}$ $C$ mean? Intuitively, the first would be a parallel process with structure $\sigma$ that produces a parallel process with structure $\sigma^{\prime}$, and the second would be a parallel process with structure $\sigma^{\prime}$ that takes a parallel process with structure $\sigma$ as input, and produces an output of type $C$. In contrast to using functions with a type such as $X \stackrel{\sigma_{1}}{\longmapsto} Y \rightarrow A \stackrel{\sigma_{2}}{\longmapsto} B$, we have so far not seen the benefits of full higher-order functions in our examples, although this is an idea that may be worth exploring in future work.

\section{Examples}

Our first two examples revisit image merge from Section 1, and quicksort from Section 3. In both cases, we show how the type system calculates the convertibility of the structured expression to a functionally equivalent parallel process, and how the convertibility proof allows the structured expression to be rewritten to the desired parallel process. The final two examples show how to use types to parallelise different algorithms, described as structured expressions. These examples show how to easily introduce parallel structure to these algorithms using our type system.

\subsection{Image Merge}

Recall that image merge basically composes two functions: mark and merge. It can be directly parallelised using different combinations of farms and pipelines.

$$
\begin{aligned}
& \operatorname{IM}_{1}(n, m)=\operatorname{PAR}_{L}(\text { FARM } n(\mathrm{FUN} \mathrm{A}) \| \text { FARM } m(\text { FUN A })) \\
& \text { imageMerge }: \operatorname{List~}(\operatorname{Img} \times \operatorname{Img}) \stackrel{\operatorname{IM}_{1}(n, m)}{\longmapsto} \operatorname{List}(\operatorname{Img} \times \operatorname{Img}) \\
& \text { imageMerge }=\operatorname{map}_{\text {List }}(\text { merge } \circ \text { mark })
\end{aligned}
$$

First, we use our annotated typing rules to produce a derivation tree with the structure of the expression (Fig. 10). The key part is the convertibility proof, that $\mathrm{MAP}_{L}(\mathrm{~A} \circ \mathrm{A}) \cong \mathrm{IM}_{1}(n, m)$. We use the decision procedure defined in Section 4 to decide the equivalence of both structures. The erase step is applied as follows:

$$
\operatorname{IM}_{1}(n, m) \rightsquigarrow{ }^{*} \operatorname{MAP}_{L}(\mathrm{~A} \odot \mathrm{A})
$$




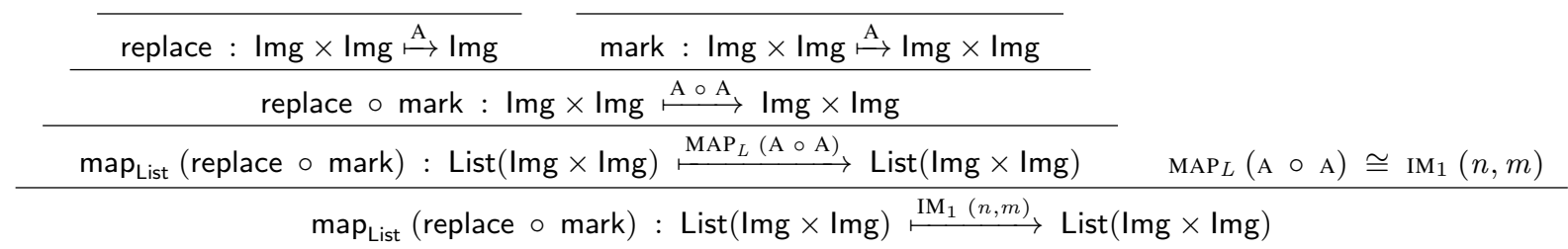

Figure 10: Typing Derivation Tree for Image Merge

The final step involves applying the decision procedure for equality of $\bar{S}$. Since the expressions are identical, this is a trivial step. We can now apply this equivalence to the original expression:

$$
\begin{aligned}
& \operatorname{map}_{\text {List }}(\text { merge } \circ \text { mark }) \rightsquigarrow * \\
& \quad \operatorname{par}_{\text {List }}(\text { farm } n(\text { fun mark }) \| \text { farm } m \text { (fun merge) })
\end{aligned}
$$

We now show an example of unification. We define $\mathrm{IM}_{2} n=$ $\operatorname{PAR}_{L}$ (- $\|$ FARM $\left.n_{-}\right)$. First, we instantiate the structure with fresh metavariables $m_{1}$ and $m_{2}$. Then, we normalise the structure. We start by applying the erase rewriting system:

$$
\operatorname{MAP}_{L}\left(m_{2}^{\prime} \circ m_{1}^{\prime}\right) \quad \delta=\left\{m_{1} \sim \mathrm{FUN} m_{1}^{\prime}, m_{2} \sim \mathrm{FUN} m_{2}^{\prime}\right\}
$$

We then apply the normalisation in $\overline{\Sigma_{s}}$, and the unification rules:

$\operatorname{MAP}_{L} \mathrm{~A} \circ \mathrm{MAP}_{L} \mathrm{~A} \sim \operatorname{MAP}_{L} m_{2}^{\prime} \circ \operatorname{MAP}_{L} m_{1}^{\prime} \Rightarrow\left\{\left\{m_{1}^{\prime} \sim \mathrm{A}, m_{2}^{\prime} \sim \mathrm{A}\right\}\right\}$

The final step is to calculate the extension of the environment $\delta$, and the set of environments that are obtained from the unification:

$$
\begin{aligned}
\Delta= & \{\delta\} \otimes\left\{\left\{m_{1}^{\prime} \sim \mathrm{A}, m_{2}^{\prime} \sim \mathrm{A}\right\}\right\}= \\
& \left\{\left\{m_{1} \sim \mathrm{FUN} m_{1}^{\prime}, m_{2} \sim \mathrm{FUN} m_{2}^{\prime}, m_{1}^{\prime} \sim \mathrm{A}, m_{2}^{\prime} \sim \mathrm{A}\right\}\right\}
\end{aligned}
$$

Applying the substitution environment in $\Delta$ to the $\operatorname{IM}_{2}(n)$, we obtain the structure PAR $_{L}$ (FUN A $\|$ FARM $_{n}$ (FUN A)).

Finally, we briefly discuss how to extend the environment further using a procedure min cost. First, min attempts further rewritings to $m_{1}$ and $m_{2}$. To ensure termination, the process stops whenever the only option is to introduce a task farm to an existing task farm structure.

$$
\begin{array}{lll}
\delta_{1}=\left\{m_{1} \sim \text { FARM } n_{1}(\text { FUN A }),\right. & \left.m_{2} \sim \text { FARM } n_{2}(\text { FUN A })\right\} \\
\delta_{2}=\left\{m_{1} \sim\right. \text { FUN A, } & & \left.m_{2} \sim \text { FARM } n_{2}(\text { FUN A })\right\} \\
\delta_{3}=\left\{m_{1} \sim \text { FARM } n_{1}(\text { FUN A }),\right. & m_{2} \sim \text { FUN A } \\
\delta_{4}=\left\{m_{1} \sim \text { FUN A },\right. & & \left.m_{2} \sim \text { FUN A }\right\}
\end{array}
$$

We will show how to select one of those structures using a simple example cost model. In future work, we will consider how to extend and formalise this cost model. A cost model provides size functions $|\sigma|$ over structures, similar to the idea of sized types [17]. We assume that all atomic functions are annotated with their cost models, $\mathrm{A}_{c}$. The cost of a structure is a function that receives a size, $s z$, and returns an estimation of its run-time in milliseconds.

In our example, we assume that $s z=[d]^{1000}$. This represents the size of 1000 pairs of images of $d$ dimensions. Arithmetic operations on $s z$ are applied to the superscript. The size function of the first stage $\left|\mathrm{A}_{c_{1}}\right|$ is the identity, since we are not modifying the images. The parameters for the number of farm workers are fixed to be those with the least cost, given some maximum number of available cores. In this example, we assume that a maximum of 24 cores are available. For $\delta_{1}$, we determine that $n_{1}=9, n=3$ and $n_{2}=5$. The values of the costs on those sizes, and the overheads of farms and pipelines $\left(\kappa_{1}\right.$ and $\left.\kappa_{2}\right)$ are given below. In the following examples, we omit these numbers and just provide the estimation for a 24-core architecture with similar overheads for farms, pipelines, divide-and-conquer and feedback.

$$
\begin{aligned}
& c_{1}[(2048 \times 2048,2048 \times 2048)]^{n}=n \times 25.11 \mathrm{~ms} \\
& c_{2}[(2048 \times 2048,2048 \times 2048)]^{n}=n \times 45.21 \mathrm{~ms} \\
& \kappa_{1}(9)=29.66 \mathrm{~ms} \quad \kappa_{1}(3 \times 5)=60.93 \mathrm{~ms} \\
& \kappa_{2}(9,3 \times 5)=114.4 \mathrm{~ms} \\
& \text { cost }\left(\delta_{1} \mathrm{IM}_{2}(n)\right) s z \\
& =\max \left\{c_{1}\left(\frac{s z}{n_{1}}\right)+\kappa_{1}\left(n_{1}\right), c_{2}\left(\frac{\left|\mathrm{A}_{c_{1}}\right|(s z)}{n \times n_{2}}\right)+\kappa_{1}\left(n \times n_{2}\right)\right\} \\
& +\kappa_{2}\left(n_{1}, n \times n_{2}\right)=3145.69 \mathrm{~ms} \\
& \operatorname{cost}\left(\delta_{2} \mathrm{IM}_{2}(n)\right) \mathrm{sz} \quad=25123.81 \mathrm{~ms} \\
& \operatorname{cost}\left(\delta_{3} \mathrm{IM}_{2}(n)\right) \mathrm{sz} \quad=3189.60 \mathrm{~ms} \\
& \text { cost }\left(\delta_{4} \mathrm{IM}_{2}(n)\right) \mathrm{sz} \quad=25123.81 \mathrm{~ms}
\end{aligned}
$$

The structure that results from applying $\delta_{1}$ is the least cost one, $\delta_{1}\left(\operatorname{IM}_{2}(3)\right)$, with $n_{1}=9$ and $n_{2}=5$.

\subsection{Quicksort}

We will now revisit quicksort and show how it can exploit a divideand-conquer parallel structure.

$$
\begin{aligned}
& \text { qsorts }: \operatorname{List}(\text { List } A) \rightarrow \operatorname{List}(\text { List } A) \\
& \text { qsorts }=\operatorname{map}_{\text {List }}\left(\text { hylo }{ }_{F A} \text { merge div }\right)
\end{aligned}
$$

In order to introduce a divide-and-conquer parallel structure, the type system needs to decide:

$$
\operatorname{MAP}_{L}\left(\mathrm{HYLO}_{F} \mathrm{AA}\right) \cong \operatorname{PAR}_{L}\left(\mathrm{DC}_{n}, F \mathrm{AA}\right)
$$

This can be achieved using a simple parallelism erasure. Consider now a slightly more complex structure:

$$
\operatorname{MAP}_{L}\left(\mathrm{HYLO}_{F} \text { A A }\right) \cong \operatorname{PAR}_{L}\left(\operatorname{FARM}_{n}-\|{ }_{-}\right)
$$

Let $m_{1}, m_{2}$ be two fresh metavariables. The parallelism erasure of the right hand side returns the following structure and substitution:

$$
\begin{array}{r}
\operatorname{PAR}_{L}\left(\text { FARM } n m_{2} \| m_{1}\right) \rightsquigarrow{ }^{*} \operatorname{MAP}_{L}\left(m_{1}^{\prime} \circ m_{2}^{\prime}\right) \\
\delta=\left\{m_{1} \sim \text { FUN } m_{1}^{\prime}, m_{2} \sim \text { FUN } m_{2}^{\prime}\right\}
\end{array}
$$

The normalisation procedure continues by normalising the left and right hand sides of the equivalence following a parallelism erasure. The left hand side is normalised by applying HYLO-SPLIT, F-SPLIT and CATA-SPLIT:

$$
\operatorname{MAP}_{L}\left(\mathrm{HYLO}_{F} \text { A A }\right) \rightsquigarrow{ }^{*} \operatorname{MAP}_{L}\left(\mathrm{CATA}_{F} \mathrm{~A}\right) \circ \operatorname{MAP}_{L}\left(\operatorname{ANA}_{F} \mathrm{~A}\right)
$$

The right hand side of the equivalence is normalised by applying F-SPLIT and CATA-SPLIT:

$$
\operatorname{MAP}_{L}\left(m_{1}^{\prime} \circ m_{2}^{\prime}\right) \rightsquigarrow{ }^{*} \mathrm{MAP}_{L} m_{1}^{\prime} \circ \operatorname{MAP}_{L} m_{2}^{\prime}
$$

The decision procedure finishes by unifying both structures, and extending the substitution $\delta$ with all possible unifications.

$$
\begin{gathered}
\operatorname{MAP}_{L}\left(\operatorname{CATA}_{F} \mathrm{~A}\right) \circ \operatorname{MAP}_{L}\left(\operatorname{ANA}_{F} \mathrm{~A}\right) \sim \operatorname{MAP}_{L} m_{1}^{\prime} \circ \mathrm{MAP}_{L} m_{2}^{\prime} \\
\Rightarrow \Delta_{1}=\left\{m_{1}^{\prime} \sim \mathrm{CATA}_{F} \mathrm{~A}, m_{2}^{\prime} \sim \mathrm{ANA}_{F} \mathrm{~A}\right\} \\
\Delta=\{\delta\} \otimes \Delta_{1}
\end{gathered}
$$


Again, by applying the only substitution in $\delta^{\prime} \in \Delta$, we select the final structure:

$$
\operatorname{PAR}_{L}\left(\operatorname{FARM}_{n}\left(\operatorname{FUN}\left(\operatorname{ANA}_{F} \mathrm{~A}\right)\right) \| \operatorname{FUN}\left(\operatorname{CATA}_{F} \mathrm{~A}\right)\right)
$$

The full proof of equivalence (@) allows us to rewrite quicksort to our desired parallel structure:

$$
\begin{aligned}
& \operatorname{map}_{\text {List }}\left(\text { hylo }_{F}{ }_{A} \text { merge div }\right) \rightsquigarrow^{*} \\
& \left.\left.\left.\quad \operatorname{par}_{\text {List }}(\text { farm } n \text { (fun (ana } F A \text { div })\right) \| \text { fun (cata } F A \text { merge }\right)\right)
\end{aligned}
$$

We can use our cost model again, where $\kappa_{3}$ is the overhead of a divide-and-conquer structure. In this example, we set the size parameter of our cost model to 1000 lists of 3,000,000 elements, and use the following structure:

$$
\begin{aligned}
& \text { qsorts : } \operatorname{List}(\text { List } A) \stackrel{\text { min cost }}{\longmapsto} \operatorname{List}(\text { List } A) \\
& \text { cost }\left(\operatorname{PAR}_{L}\left(\mathrm{DC}_{n, F} \mathrm{~A}_{c_{1}} \mathrm{~A}_{c_{2}}\right)\right) s z \\
& =\max \left\{\operatorname { m a x } _ { 1 \leq i \leq n } \left\{c_{2}\left(\left|\mathrm{~A}_{c_{2}}\right|^{i} s z\right)\right.\right. \\
& , \operatorname{cost}\left(\mathrm{HYLO}_{F} \mathrm{~A}_{c_{1}} \mathrm{~A}_{c_{2}}\right)\left(\left|\mathrm{A}_{c_{2}}\right|^{n} s z\right) \\
& \left., \max _{1 \leq i \leq n}\left\{c_{1}\left(\left|\mathrm{~A}_{c_{1}}\right|^{i}\left|\mathrm{~A}_{c_{2}}\right|^{n} s z\right)\right\}\right\}+\kappa_{3}(n)=42602.72 \mathrm{~ms} \\
& \operatorname{cost}\left(\operatorname{PAR}_{L}\left(\operatorname{FARM}_{n}\left(\operatorname{FUN}\left(\operatorname{ANA}_{L} \mathrm{~A}_{c_{2}}\right)\right) \|\left(\operatorname{FUN}\left(\operatorname{CATA}_{L} \mathrm{~A}_{c_{1}}\right)\right)\right)\right) s z \\
& =27846.13 \mathrm{~ms} \\
& \text { cost }\left(\operatorname{PAR}_{L}\left(\operatorname{FARM}_{n}\left(\operatorname{FUN}\left(\operatorname{HYLO}_{F} \mathrm{~A}_{c_{1}} \mathrm{~A}_{c_{2}}\right)\right)\right)\right) s z \\
& =32179.77 \mathrm{~ms} \\
& \text {... }
\end{aligned}
$$

Since the most expensive part of the quicksort is the divide, and flattening a tree is linear, the cost of adding a farm to the divide part is less than using a divide-and-conquer skeleton for this example.

\subsection{N-Body Simulation}

N-Body simulations are widely used in astrophysics. They comprise a simulation of a dynamic system of particles, usually under the influence of physical forces. The Barnes-Hut simulation recursively divides the $n$ bodies storing them in a Octree, or a 8-ary tree. Each node in the tree represents a region of the space, where the topmost node represents the whole space and the eight children the eight octants of the space. The leaves of the tree contain the bodies. Then, the cumulative mass and center of mass of each region of the space are calculated. Finally, the algorithm calculates the net force on each particular body by traversing the tree, and updates its velocity and position. This process is repeated for a number of iterations. We will here abstract most of the concrete, well known details of the algorithm, and present its high-level structure, using the following types and functions:

$$
\begin{array}{ll}
C & =\mathbb{Q} \times \mathbb{Q} \\
F A B & =A+C \times B^{8} \\
G A & =F \text { Body } \\
\text { Octree } & =\mu G \\
\text { insert } & : \text { Body } \times \text { Octree } \rightarrow \text { Octree }
\end{array}
$$

Since this algorithm also involves iterating for a fixed number of steps, we define iteration as a hylomorphism. We assume that the combinator + (Fig. 1) is also defined in $\Sigma_{s}$. Additionally, we assume a primitive combinator, that tests a predicate on a value, $(\cdot ?):(A \rightarrow \mathrm{Bool}) \rightarrow A \rightarrow A+A$.

$$
\begin{aligned}
& \text { LOOP : } \Sigma \rightarrow \Sigma \\
& \text { LOOP } \sigma=\operatorname{HYLO}_{(+)}(\mathrm{ID} \nabla \sigma)((\mathrm{A}+(\mathrm{A} \triangle(\mathrm{A} \circ \mathrm{A}))) \circ(\mathrm{A} \circ \mathrm{A} ?)) \\
& \operatorname{loop}_{A}:(A \stackrel{m}{\longmapsto} A) \rightarrow A \times \mathbb{N} \stackrel{\text { LOOP } m}{\longmapsto} A \\
& \operatorname{loop}_{A} s= \\
& \text { hylo }_{(A+)}(\text { id } \nabla s) \\
& \left(\left(\pi_{1}+\left(\pi_{1} \triangle\left((-1) \circ \pi_{2}\right)\right)\right) \circ\left((==0) \circ \pi_{2}\right) ?\right)
\end{aligned}
$$

This example uses some additional functions: calcMass annotates each node with the total mass and centre of mass; dist distributes the octree to all the bodies, so that each can independently calculate its forces and update the velocity and position; calcForce calculates the force of one body; and move updates the velocity and position of the body.

$$
\begin{aligned}
& \text { calcMass : } G \text { Octree } \rightarrow G \text { Octree } \\
& \text { dist : Octree } \times \text { List Body } \\
& \quad \rightarrow L(\text { Octree } \times \text { Body })(\text { Octree } \times \text { List Body })
\end{aligned}
$$

The algorithm is:

$$
\begin{aligned}
& \text { nbody : List Body } \times \mathbb{N} \stackrel{\text { LOOP } \sigma}{\longrightarrow} \text { List Body } \\
& \text { nbody }=\operatorname{loop~}\left(\operatorname{ana}_{L}(L(\text { move } \circ \text { calcForce }) \circ \text { dist })\right. \\
& \left.\quad \circ\left(\left(\text { cata }_{G}\left(\text { in }_{G} \circ \text { calcMass }\right) \circ \text { cata }_{L} \text { insert }\right) \triangle \text { id }\right)\right)
\end{aligned}
$$

Since the LOOP defines a fixed structure, we do not allow any rewriting that changes this structure. However, note that our type system still enables some interesting rewritings. In particular, the structure of the loop body is:

$$
\sigma=\operatorname{ANA}_{L}(L(\mathrm{~A} \circ \mathrm{A}) \circ \mathrm{A}) \circ\left(\mathrm{CATA}_{G}(\mathrm{IN} \circ \mathrm{A}) \circ \mathrm{CATA}_{L} \mathrm{~A}\right) \triangle \mathrm{ID}
$$

The normalised structure reveals more possibilities for introducing parallelism:

$\sigma=\operatorname{MAP}_{L} \mathrm{~A} \circ \mathrm{MAP}_{L} \mathrm{~A} \circ \mathrm{ANA}_{L} \mathrm{~A} \circ\left(\operatorname{CATA}_{G}(\mathrm{IN} \circ \mathrm{A}) \circ \mathrm{CATA}_{L} \mathrm{~A}\right) \triangle \mathrm{ID}$

After normalisation, this structure is equivalent to:

$$
\sigma=\operatorname{PAR}_{L}(\mathrm{FUN}(\mathrm{A} \circ \mathrm{A})) \circ_{-}
$$

The structure makes it clear that there are many possibilities for parallelism using farms and pipelines. As before, parallelism can be introduced semi-automatically using a cost model. For example, setting the input size to 20,000 bodies:

$$
\begin{aligned}
& \sigma=\operatorname{PAR}_{L}\left(\text { FARM } n_{-} \|-\right) \circ_{-} \\
& \sigma^{\prime}=\operatorname{PAR}_{L}(\min \operatorname{cost}(-\|-)) \circ_{-} \\
& \operatorname{cost}\left(\mathrm{FUN} \mathrm{A}_{c_{1}} \| \mathrm{FUN} \mathrm{A}_{c_{2}}\right) s z=310525.67 \mathrm{~ms} \\
& \operatorname{cost}\left(\mathrm{FARM}_{6}\left(\mathrm{FUN} \mathrm{A}_{c_{1}}\right) \|\left(\mathrm{FUN} \mathrm{A}_{c_{2}}\right)\right) s z=55755.43 \mathrm{~ms} \\
& \operatorname{cost}\left(\mathrm{FUN} \mathrm{A}_{c_{1}} \| \mathrm{FARM}_{1}\left(\mathrm{FUN} \mathrm{A}_{c_{2}}\right)\right) s z=310525.67 \mathrm{~ms} \\
& \operatorname{cost}\left(\mathrm{FARM}_{20}\left(\mathrm{FUN} \mathrm{A}_{c_{1}}\right) \| \mathrm{FARM}_{4}\left(\mathrm{FUN} \mathrm{A}_{c_{2}}\right)\right) s z=15730.46 \mathrm{~ms}
\end{aligned}
$$

\subsection{Iterative Convolution}

Image convolution is also widely used in image processing applications. We assume the type Img of images, the type Kern of kernels, the functor $F A B=A+B \times B \times B \times B$, and the following functions. The split function splits an image into 4 sub-images with overlapping borders, as required for the kernel. The combine function concatenates the sub-images in the corresponding positions. The kern function applies a kernel to an image. Finally, the finished function tests whether an image has the desired properties, in which case the computation terminates. We can represent image convolution on a list of input images as follows:

$$
\begin{aligned}
& \text { conv : Kern } \rightarrow(\text { List Img } \stackrel{\sigma}{\mapsto} \text { List Img }) \\
& \text { conv } k= \\
& \text { map }_{\text {List }}\left(\text { (iter }_{\text {Img }}(\text { finished? o hylo }\right. \\
& F \\
& (\text { combine } \circ F(\text { kern } k)) \\
& (\text { split } k)))
\end{aligned}
$$


The structure of conv is equivalent to a feedback loop, which exposes many opportunities for parallelism. Again, we assume a suitable cost model, and the estimations are given for 1000 images, of size $2048 \times 2048$.

$$
\begin{aligned}
& \sigma=\operatorname{PAR}_{L}\left(\mathrm{FB}\left(\mathrm{DC}_{n, L, F}(\mathrm{~A} \circ F \mathrm{~A}) \mathrm{A} \|-\right)\right) \\
& =\operatorname{PAR}_{L}\left(\mathrm{FB}\left(\operatorname{FARM} n_{-}\|-\|-\right)\right) \\
& =\min \operatorname{cost}\left(\operatorname{PAR}_{L}\left(\mathrm{FB}\left({ }_{-} \|-\right)\right)\right) \\
& =\ldots \\
& \operatorname{cost}\left(\operatorname{PAR}_{L}\left(\mathrm{FB}\left(\mathrm{A}_{c_{1}} \| \mathrm{A}_{c_{2}}\right)\right)\right) s z= \\
& \sum_{1 \leq i,\left|\mathrm{~A}_{c_{1}} \| \mathrm{A}_{c_{2}}\right|^{i} s z>0} \operatorname{cost}\left(\mathrm{A}_{c_{1}} \| \mathrm{A}_{c_{2}}\right)\left(\left|\mathrm{A}_{c_{1}} \| \mathrm{A}_{c_{2}}\right|^{i} s z\right) \\
& =20923.02 \mathrm{~ms} \\
& \text { cost }\left(\operatorname{PAR}_{L}\left(\mathrm{FB}\left(\mathrm{FARM}_{4}\left(\mathrm{FUN} \mathrm{A}_{c_{1}}\right) \|\left(\mathrm{FUN} \mathrm{A}_{c_{2}}\right)\right)\right)\right) s z \\
& =6649.55 \mathrm{~ms} \\
& \operatorname{cost}\left(\operatorname{PAR}_{L}\left(\mathrm{FB}\left(\mathrm{FUN} \mathrm{A}_{c_{1}} \| \mathrm{FARM}_{1}\left(\mathrm{FUN} \mathrm{A}_{c_{2}}\right)\right)\right)\right) s z \\
& =20923.02 \mathrm{~ms} \\
& \text { cost }\left(\operatorname{PAR}_{L}\left(\mathrm{FB}\left(\mathrm{FARM}_{14}\left(\mathrm{FUN} \mathrm{A}_{c_{1}}\right) \| \operatorname{FARM}_{4}\left(\mathrm{FUN} \mathrm{A}_{c_{2}}\right)\right)\right)\right) s z \\
& =2694.30 \mathrm{~ms} \\
& \ldots
\end{aligned}
$$

Collectively, our examples have demonstrated the use of our techniques for all the parallel structures we have considered, showing that we can easily and automatically introduce parallelism according to some required structure, while maintaining the functional equivalence with the original form.

\section{Related Work}

There have been some previous treatments of parallelism using types, but these deal only with sizes and productivity. One line of work is using sized types [17] to internalise a notion of sizes of streaming data into a type system. This has been extended to a small number of skeletons in the Eden language [27]. While types were useful to prove the termination and productivity of Eden skeletons, our own work focuses on the different, but important, properties of semantic equivalence and cost. The expressive power of hylomorphisms for parallel programming was first explored by Fischer and Gorlatch [4], who showed that a programming language based on catamorphisms and anamorphisms is Turing-universal. The idea of using hylomorphisms for parallel programming also appears in Morihata's work [24]. Morihata explores a theory for developing parallelisation theorems based on the third homomorphism theorem and shortcut fusion, and generalises it to hylomorphisms. In contrast, our work directly exploits the properties of hylomorphisms, in order to choose a suitable parallel skeleton implementation for hylomorphisms. Both lines of work are therefore orthogonal, and we can potentially benefit from Morihata's results.

Deriving parallel implementations from small, simple specifications has been widely studied. The third homomorphism theorem, list homomorphisms, and the Bird-Meertens Formalism are amongst the many techniques that have been explored [12, 15, 16, $18,21,23,25,29-31]$. The third homomorphism theorem states that if a function can be written both as a left fold and a right fold, then it can also be evaluated in a divide-and-conquer manner [9]. This theorem has been widely used for parallelism $[2,6,8,11,19$, $24,26]$. The majority of this work enables suitable automation and derivation of efficient parallel implementations. Our work differs in that we allow part of the parallel structure to be chosen in a semi-automated way. This adds flexibility, enabling a parallel implementation to be changed quickly and easily by changing only a single type annotation. One possible extension of our work is to include some automatic transformations derived from the third homomorphism theorem. By parameterising our type system over some cost function on parallel structures, we smoothly integrate the introduction of parallelism with the ability to reason about the run- time behaviour of the parallel program. Skillicorn and Cai [32] have previously shown the utility of such an integration of a cost calculus with derivational software development, illustrating the approach for the Bird-Meertens theory of lists. We take this approach one step further by using a more general equational theory based on hylomorphisms. Moreover, our type-based approach introduces new benefits, by providing a mechanism for specifying new parallel structures whose denotational semantics can be described as a composition of hylomorphisms.

Finally, in a practical setting, Steuwer et al [33] generate highperformance OpenCL code from a high-level specification by applying a simple set of rewrite rules, and using Monte-Carlo search to traverse the corresponding search space to find an implementation. Our semi-automated approach provides a way to narrow down this search space, while using cost models to automate the rest. Our approach is in a sense more general, since we allow our parallel structures to be easily extended. However, we could benefit from exploiting their work in GPU-specific rewriting rules and skeletons.

\section{Conclusions}

This paper has introduced a new type-based approach for representing and reasoning about the structure of parallel programs represented as algorithmic skeletons, the first ever treatment of parallelism at the type level that combines reasoning about program equivalences and cost. Crucially, our type system is capable of reasoning about both the functional and the non-functional properties of a parallel program. Given a cost model and the underlying equational theory, we have shown how we can take rational choices at the type level between alternative parallel implementations by appropriately instantiating and transforming high-level parallelism abstractions. This avoids the usual static analysis approach that separates analysis from program. In particular, all transformations are performed internally by the type checker and we ensure the preservation of the underlying functional behaviour simply by construction. It also opens the door to further safe type-level program manipulations, for example. A key aspect of our approach is the use of hylomorphisms, combinations of catamorphisms and anamorphisms (or fold/unfold operations), as a single, unifying parallel structure. In this paper we have used hylomorphisms to capture many common patterns of parallelism that are found in the literature, including inter alia task farms, pipelines, divide-and-conquer and dynamic feedback. As we have shown in our examples, this single construct is surprisingly powerful, providing a system of canonical representations that is easy to understand and to transform.

A number of obvious extensions can be made to this work. Firstly, there are a few forms of parallel pattern that we have not yet considered: map (reduce) and fold are clearly instances of hylomorphisms, but stencil and bulk synchronous parallel patterns, for example, may require deeper thought. Secondly, more sophisticated and accurate cost models are both possible and desirable, including ones that consider e.g. the sizes of data structures. The model we have shown here is, however, more precise and realistic than e.g. typical PRAM models that are widely used in parallelism theory. Thirdly and finally, we have only shown a static analysis here. However, type-based approaches can freely admit dynamic analyses as well. We intend to explore this in future in order to obtain even more general and flexible analyses.

\section{Acknowledgements}

This work has been partially supported by the EU H2020 grant "RePhrase: Refactoring Parallel Heterogeneous Resource-Aware Applications - a Software Engineering Approach" (code 644235), and by EPSRC grant EP/M027317/1 " $\mathrm{C}^{3}$ : Scalable \& Verified Shared Memory via Consistency-directed Cache Coherence". 


\section{References}

[1] F. Baader and T. Nipkow. Term Rewriting and All That. Cambridge University Press, 1998.

[2] Y.-Y. Chi and S.-C. Mu. Constructing List Homomorphisms from Proofs. In Proc. APLIAS '11: Asian Symposium on Programming Languages \& Systems, pages 74-88. 2011.

[3] M. I. Cole. Algorithmic Skeletons: Structured Management of Parallel Computation. Pitman, London, 1989.

[4] J. Fischer and S. Gorlatch. Turing Universality of Recursive Patterns for Parallel Programming. Parallel Processing Letters, 12(02):229_ 246, 2002.

[5] M. M. Fokkinga and E. Meijer. Program Calculation Properties of Continuous Algebras. Technical Report, CWI, 1991.

[6] A. Geser and S. Gorlatch. Parallelizing Functional Programs by Generalization. Journal of Functional Programming (JFP), 9(06): 649-673, 1999.

[7] N. Ghani. $\beta \eta$-Equality for Coproducts. In Proc. International Conf. on Typed Lambda Calculi and Applications, pages 171-185. 1995.

[8] J. Gibbons. Computing Downwards Accumulations on Trees Quickly. Theoretical Computer Science, 169(1):67-80, 1996.

[9] J. Gibbons. The Third Homomorphism Theorem. Journal of Functional Programming (JFP), 6(4):657-665, 1996.

[10] J. Gibbons. Calculating Functional Programs. In Algebraic and Coalgebraic Methods in the Mathematics of Program Construction, pages 151-203. 2002.

[11] S. Gorlatch. Extracting and Implementing List Homomorphisms in Parallel Program Development. Science of Computer Programming, 33(1): 1 - 27, 1999.

[12] S. Gorlatch and C. Lengauer. Parallelization of Divide-and-Conquer in the Bird-Meertens Formalism. Formal Aspects of Computing, 7(6): 663-682, 1995.

[13] T. Hardin. Confluence Results for the Pure Strong Categorical Logic CCL. $\lambda$-calculi as Subsystems of CCL. Theoretical Computer Science, 65(3):291-342, 1989.

[14] Y. Hayashi and M. Cole. Static Performance Prediction of Skeletal Parallel Programs. Parallel Algorithms and Applications, 17(1):5984, 2002.

[15] Z. Hu, H. Iwasaki, and M. Takechi. Formal Derivation of Efficient Parallel Programs by Construction of List Homomorphisms. ACM Transactions on Programming Languages and Systems (TOPLAS), 19 (3):444-461, 1997.

[16] Z. Hu, M. Takeichi, and W.-N. Chin. Parallelization in Calculational Forms. In Proc. POPL '98: 25th ACM Symposium on Principles of Programming Languages, pages 316-328, 1998.

[17] J. Hughes, L. Pareto, and A. Sabry. Proving the Correctness of Reactive Systems Using Sized Types. In Proc. POPL '96: 23rd ACM Symposium on Principles of Programming Languages, pages 410 423, 1996.

[18] G. Keller and M. Chakravarty. Flattening Trees. In Proc. Euro-Par '98: European Conference on Parallelism, pages 709-719. 1998.
[19] Y. Liu, Z. Hu, and K. Matsuzaki. Towards Systematic Parallel Programming over Mapreduce. In Proc. Euro-Par 2011: European Conference on Parallelism, pages 39-50. 2011.

[20] O. Lobachev and R. Loogen. Estimating Parallel Performance, a Skeleton-Based Approach. In Proc. HLPA'10: Intl workshop on Highlevel Parallel Prog. and Appls., pages 25-34, 2010.

[21] K. Matsuzaki, Z. Hu, and M. Takeichi. Towards Automatic Parallelization of Tree Reductions in Dynamic Programming. In Proc. SPAA 2006: Symposium on Parallelism in Algorithms and Architecture, pages 39-48, 2006.

[22] E. Meijer, M. Fokkinga, and R. Paterson. Functional Programming with Bananas, Lenses, Envelopes and Barbed Wire. In Proc. ICFP '91: ACM Conf. on Functional Programming, pages 124-144, 1991.

[23] J. Misra. Powerlist: A Structure for Parallel Recursion. ACM Transactions on Programming Languages and Systems (TOPLAS), 16(6): 1737-1767, 1994.

[24] A. Morihata. A Short Cut to Parallelization Theorems. In Proc. ICFP 2013: 18th ACM Conf. on Functional Programming, pages 245-256, 2013.

[25] A. Morihata and K. Matsuzaki. Automatic Parallelization of Recursive Functions using Quantifier Elimination. In Proc. FLOPS '10: Functional and Logic Programming, pages 321-336. 2010.

[26] K. Morita, A. Morihata, K. Matsuzaki, Z. Hu, and M. Takeichi. Automatic Inversion Generates Divide-and-Conquer Parallel Programs. In Proc. PLDI '07: ACM Conf. on Programming Language Design and Implementation, pages 146-155, 2007.

[27] R. Peña and C. Segura. Sized Types for Typing Eden Skeletons. In Proc. IFL '01: Intl. Symposium on Implementation of Functional Languages, pages 1-17, 2001.

[28] F. A. Rabhi and S. Gorlatch. Patterns and Skeletons for Parallel and Distributed Computing. Springer, 2003.

[29] J. H. Reif. Synthesis of Parallel Algorithms. Morgan Kaufmann, 1993.

[30] D. B. Skillicorn. Models for Practical Parallel Computation. International Journal of Parallel Programming, 20(2):133-158, 1991.

[31] D. B. Skillicorn. The Bird-Meertens Formalism as a Parallel Model. Springer, 1993.

[32] D. B. Skillicorn and W. Cai. A Cost Calculus for Parallel Functional Programming. J. Parallel Distrib. Comput., 28(1):65-83, 1995.

[33] M. Steuwer, C. Fensch, S. Lindley, and C. Dubach. Generating Performance Portable Code Using Rewrite Rules. In Proc ICFP 2015: 20th ACM Conf. on Functional Prog. Lang. and Comp. Arch., pages 205-217, 2015.

[34] J. E. Stoy. Denotational Semantics: The Scott-Strachey Approach to Programming Language Theory. MIT Press, Cambridge, MA, USA, 1977. ISBN 0262191474.

[35] H. Yokouchi. Church-Rosser Theorem for a Rewriting System on Categorical Combinators. Theoretical Computer Science, 65(3):271290, 1989. 